\title{
A Toolbox of Metrology-based Techniques for Optical System Alignment
}

SPIE Optics \& Photonics

San Diego, CA August 28, 2016

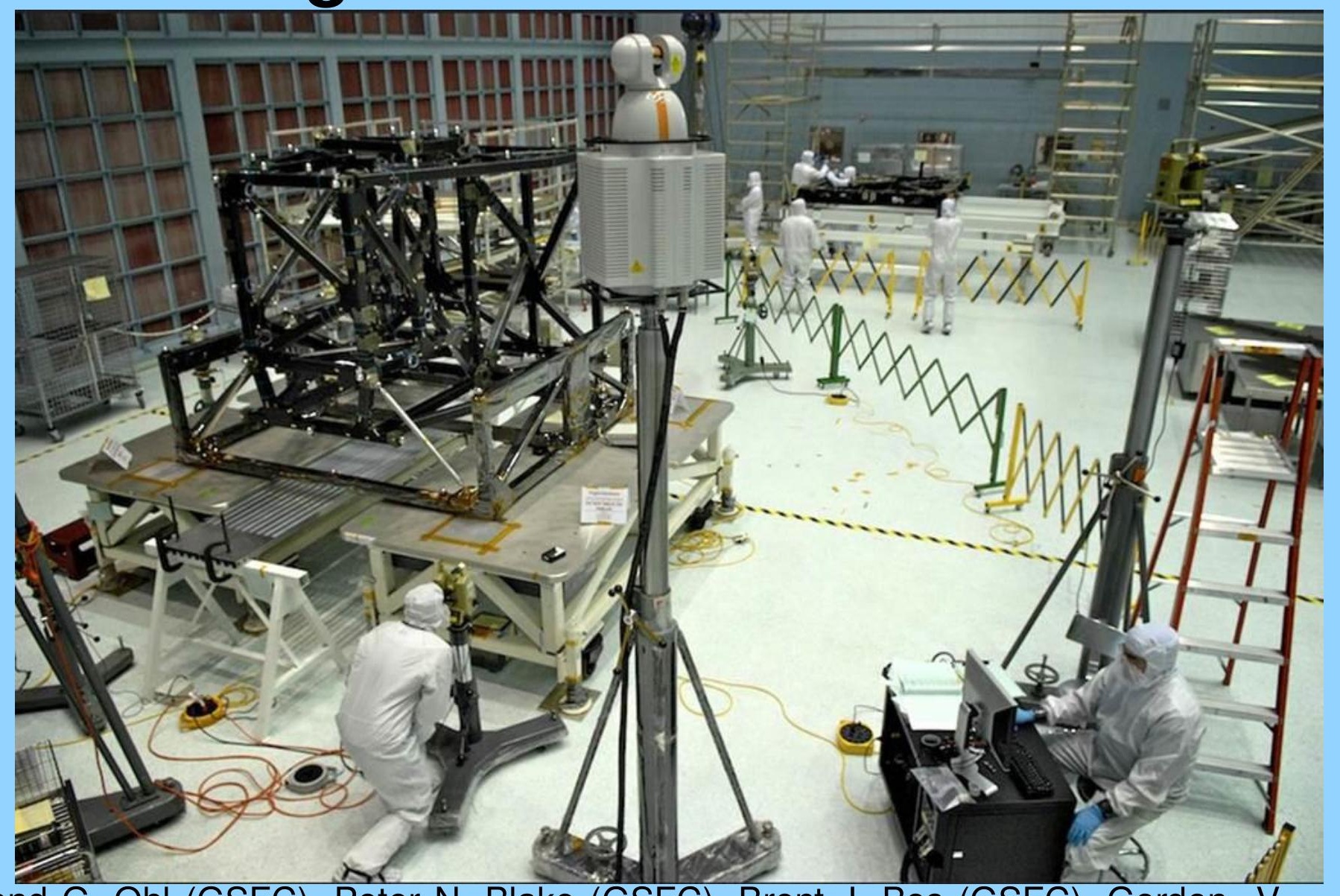

Phillip Coulter (GSFC), Raymond G. Ohl (GSFC), Peter N. Blake (GSFC), Brent J. Bos (GSFC), Gordon V. Casto (GSFC), William L. Eichhorn (GESI), Jeffrey S. Gum (GSFC), Theodore J. Hadjimichael (GSFC), John G. Hagopian (JHEL), Joseph E. Hayden (SSC), Samantha L. Hicks (GSFC), Dave Kubalak (GSFC), Kyle F. Mclean (PEMSL), Joseph McMann (SLI), Kevin Redman (SLI), Greg Wenzel (SLI), Jerrod Young (GSFC) 
I. Introduction

II. Instruments

III. Analytical tools

IV. Applications

V. Conclusion 
- The Optics branch is active in all phases of instrument and optical technology development

- Design

- Fabrication

- Components

- Optical alignment, integration and test-optical metrology

- Wavefront sensing

- GSFC has significant heritage in building and aligning spacecraft and space flight optical instruments

- NASA GSFC Optics branch involved in instrument building and optical metrology since the 1960s

- Spacecraft and instrument-level alignment, integration and testing (AI\&T)

- Optical component through system level characterization and alignment

- Environmental testing

- Mechanical system and optical alignment and verification

- Pre-launch checkout - optical end-to-end testing, alignment checks to spacecraft, etc.

- Recent relevant GSFC spacecraft alignment and space flight optical instrument projects

- JWST ISIM, OTIS, and I\&T

- HST I\&T and IVT

- OSIRIS-Rex/OVIRS

- Spitzer/IRAC

- DSCVRI\&T

- Many sounding rocket and balloon instruments
- Cassini/CIRS

- Landsat/TIRS

- IceSat/ATLAS

- IRMOS

- WMAP

- COBE 


\section{NASA GSFC Contributions to Diverse Mission Portfolio}
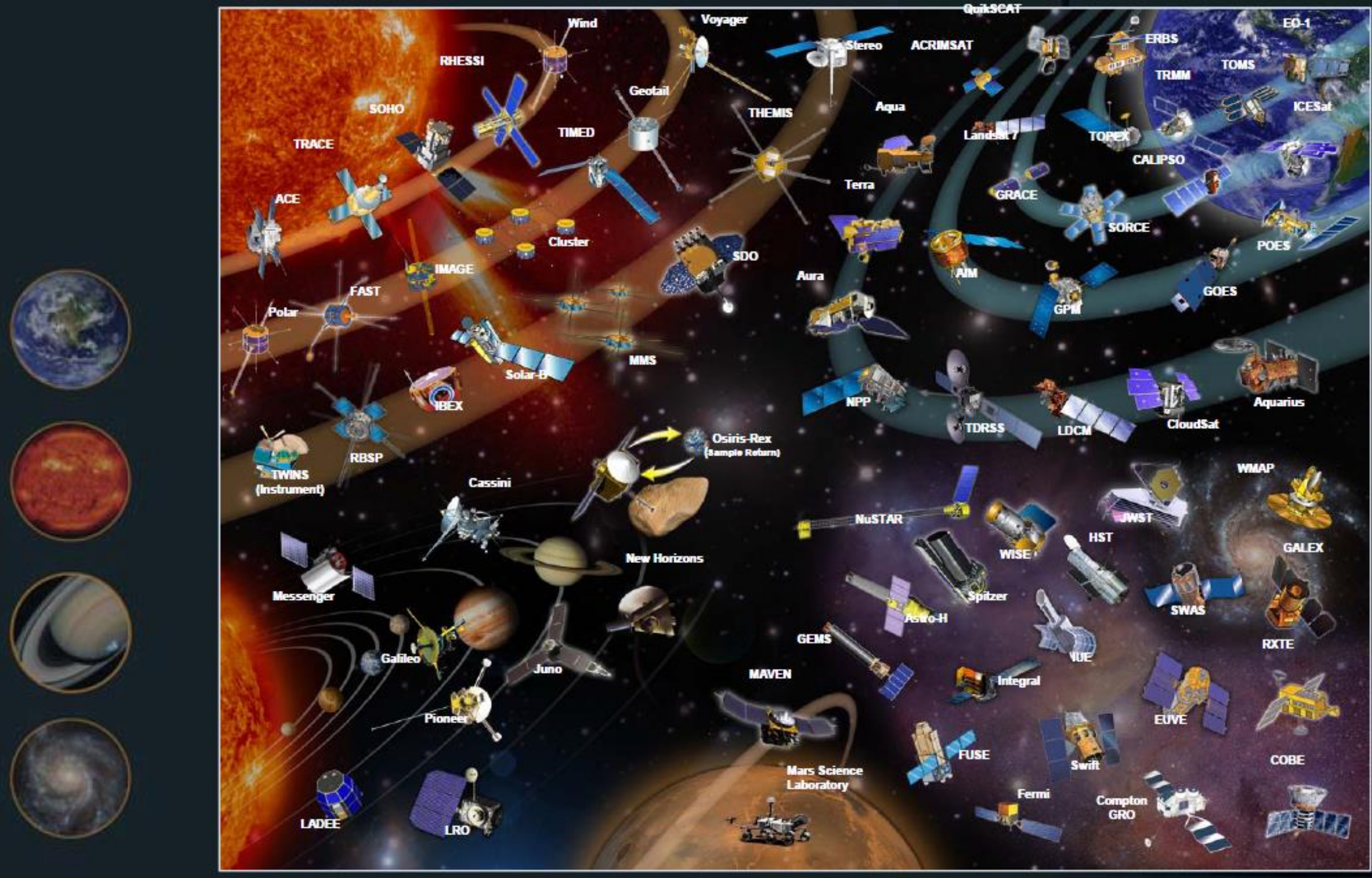


\section{Introduction}

- Provide an overview of some modern, optical/optomechanical measurement capabilities in a "toolbox" format

- Show how metrology instruments that are typically used for large-volume applications can be also used for optical alignment

- We do not discuss interferometry or wavefront sensing and similar, optical-only techniques

- those subjects are broad and well treated by others

- we focus on techniques and instruments that are less used by optical engineers and usually not covered in classical optical engineering curricula

- techniques that would be employed to accomplish alignment before high-precision optical testing/verification, like interferometry or image testing

- The concept of using these metrology instrument for alignment involves:

- Establish and measure a fixed, stable coordinate system (CS) to bench or structure

- CS is based on measurable features that are important to an optical system and transferred to metrology references that are modeled in CAD, FEM, and ray trace software

- Metrology at the component level, followed by work at the subsystem and system level

- Alignment of components to the system is achieved nominally based on nominal CAD and an optical design

- Optomechanical work is followed by optical testing for verification and/or tweaking to achieve tighter alignment and performance (interferometry, wavefront sensing, other image-based techniques)

- This methodology can simplify and speed up the alignment process --- i.e., reduce iterations.....

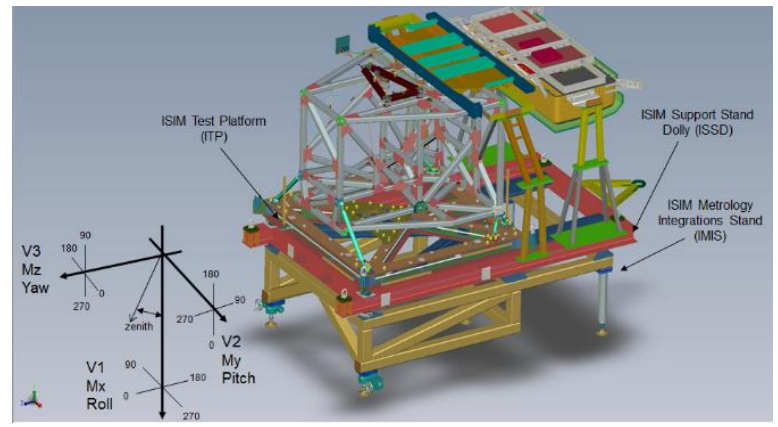


- Wide variety of measurement instruments available

- Laser Trackers (LT)

- Laser Radar (LR)

- Theodolites

- Cathetometer

- CMM with variety of probe types

- Instruments can be classified as
- Measurement arms (portable CMM)

- Theodolites

- Total Stations

- Point source microscope

- Heritage optical tools --- invaluable

- Contact vs. non-contact to the article under test

- Point-like (X, Y, Z) vs. angular (i.e., vector) measurements

- Instruments discussed are not limited to the manufacturers shown in the following examples --- specific instruments and manufacturers mentioned or shown do not constitute an endorsement by NASA --- many manufacturers and models function in a similar manner and would produce similar results --- we encourage the reader to study all available options
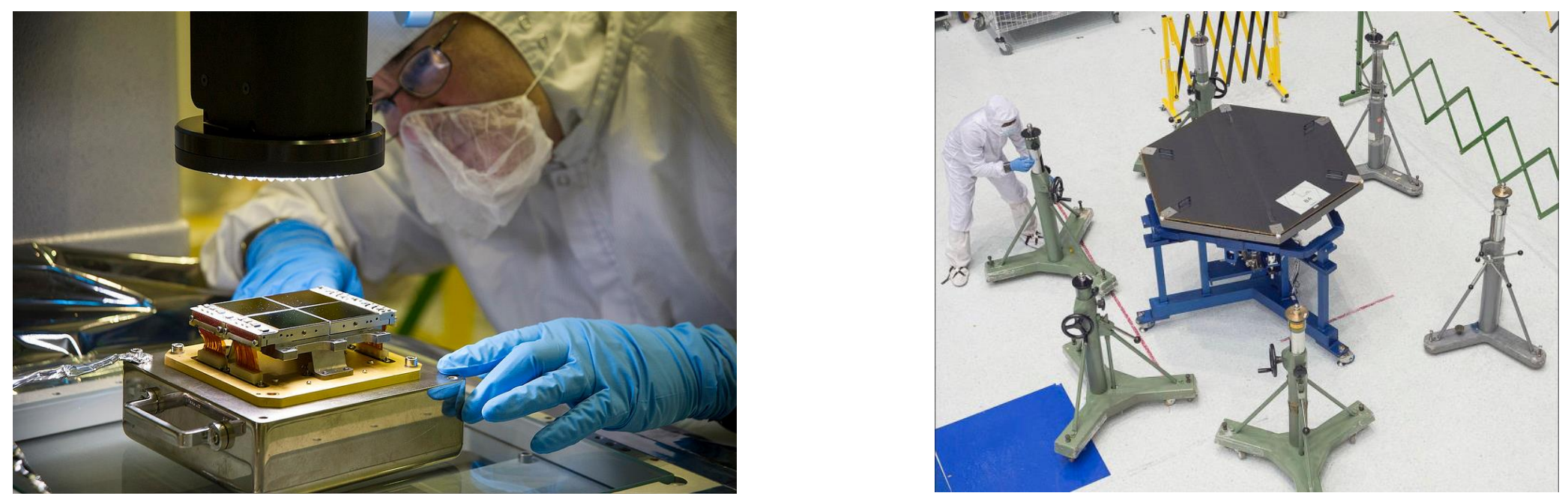


\section{Laser trackers}

the LR

- Uncertainty $\sim<0.025 \mathrm{~mm} / \mathrm{meter}$ (1-sigma)

- Some LTs may be used with probe, scanners and other accessories

- May also be used to track hardware during "blind" precision integration activities

- Can be used to track an SMR for live position feedback

- Surfaces are measured by dragging or touching the SMR in several locations on the test object

- Can be used to measure flat mirror orientation and position via "direct and through" technique ${ }^{3}$ (uncertainties on the order of 10-15 arcsec)

- Some LT models can measure flat mirror normals directly

- Advantages:

- Large measurement volume

- High accuracy

- Very flexible instrument

- Multiple attachment available for some models

- Tracks a moving target

- Disadvantages:

- Must have straight line of sight to target

1. Burge, J. et al., "Use of a commercial laser tracker for optical alignment," Proc. SPIE 6676, 2007

- Requires contact when installing SMR

- Requires $>1$ operator usually
SMR \& nest

Laser scanner attachment

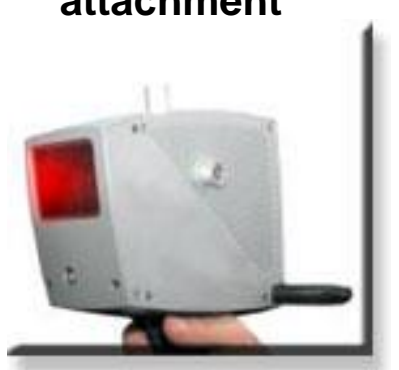

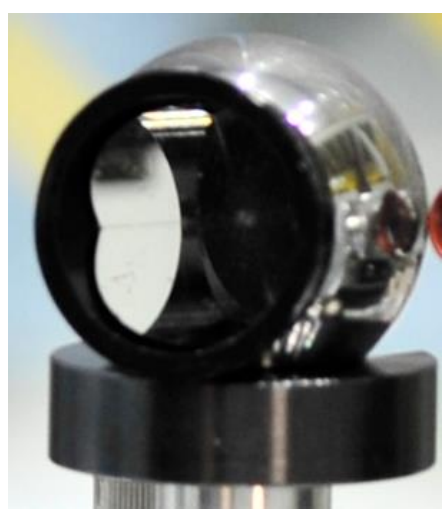

2. New River Kinematics, Inc., Williamsburg, Virginia, www.kinematics.com 3. S. Sandwith \& R. Predmore, "Real-time 5-micron Uncertainty with Laser Tracking Interferometer Systems using Weighted Trilateration," New River Kinematics, Inc. Williamsburg, Va 
- Laser radar ${ }^{1}$ (LIDAR) is used to measure targets and surfaces

- Operated with Spatial Analyzer software, USMN-compatible

- Its target is diffuse surface (mechanical surface; matt finish), reflective tooling ball, specular mirror, or high-quality tooling hole; also useful for specular, optical surfaces

- Uncertainty $\sim<0.025 \mathrm{~mm} /$ meter (1-sigma)

- Laser Radar scans much faster than Laser Tracker with TScan or tramming

- At ambient, used for:

- Prescription and alignment measurement for large optics (radius, aperture, etc.)

- Envelope scans (hardware critical clearances)

- Tooling ball targets on large and small assemblies

- Advantages:

- Non-contact scanning capabilities

- Uses TBs which cost less than SMRs

- Large measurement volume

- Less human interaction with the test article

- Requires only 1 operator

- Disadvantages:

- Requires straight line of sight to target

- Cannot track a moving target

- Expensive

1. Metris USA, Inc., Brighton, Michigan, www.metris.com
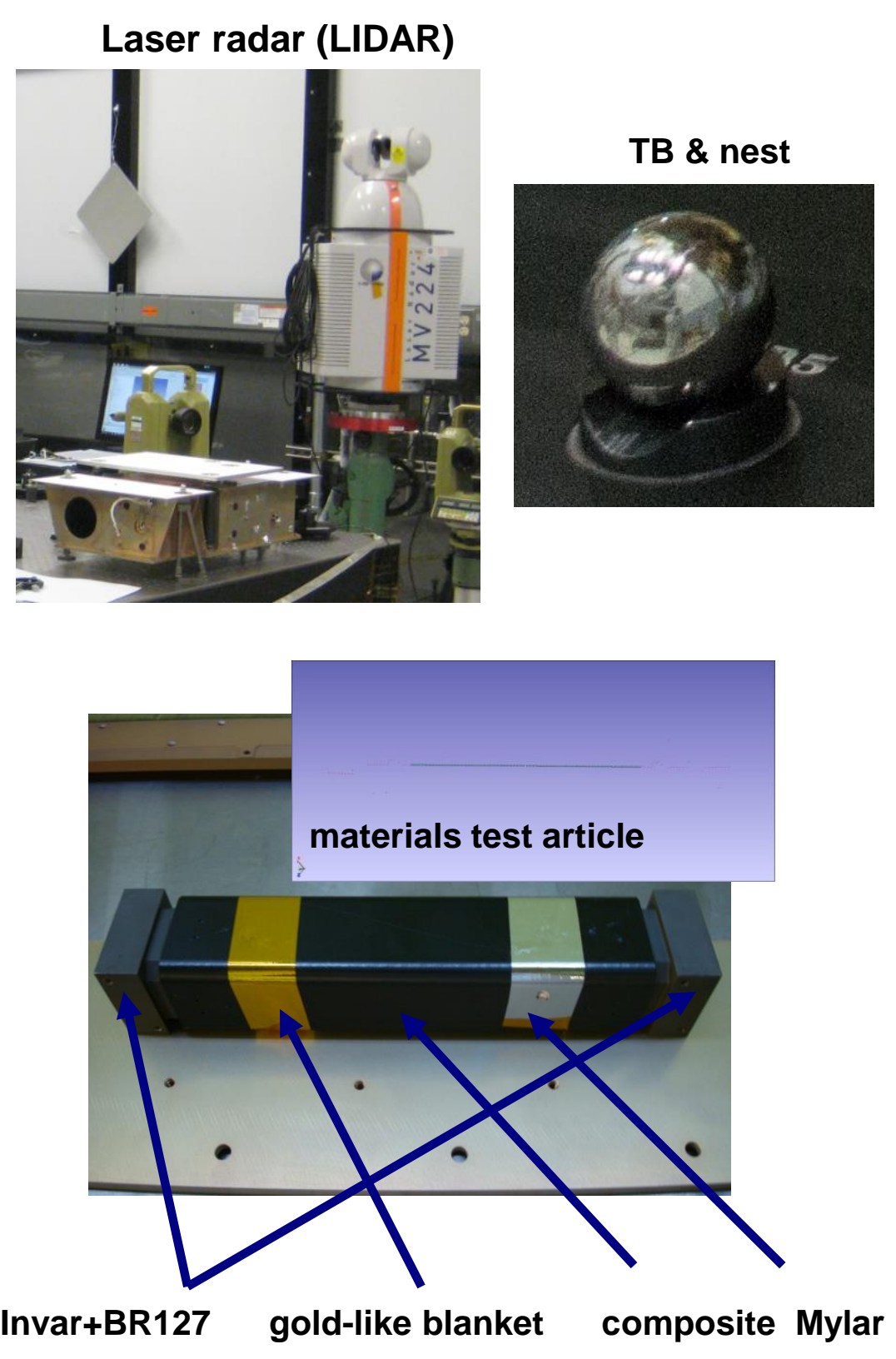


\section{Measurement Arms}

- Primarily a contact measurement system, but scanner heads available

- Available in various arm lengths

- Can measure numerous features such as TBs, SMRs, holes, and surfaces

- Advantages:

- Does not require direct line of sight to target

- Portable

- Multiple probe tips available with non-contact options

- Disadvantage:

- Measurement volume limited to arm length

- Accuracy not as good a LT and LR systems

- Measurements are made in close proximity to hardware

- Uncertainty is a complex function of distance from fixed base

\section{Total Stations}

- Theodolite with built in distance measuring laser

- Capable of measuring angle and point-like data

- Non-contact instrument (except SMR measurement)

- Angular measurement uncertainties same as theodolite

- Range uncertainty based on target type and distance

- Target types: SMR, retroreflector, any visible target measurable by the laser

- Advantages:

- Combined capability of angle and point-like measurements

- Small and portable

- Disadvantages:

- Range accuracy not as good as LR and LT systems
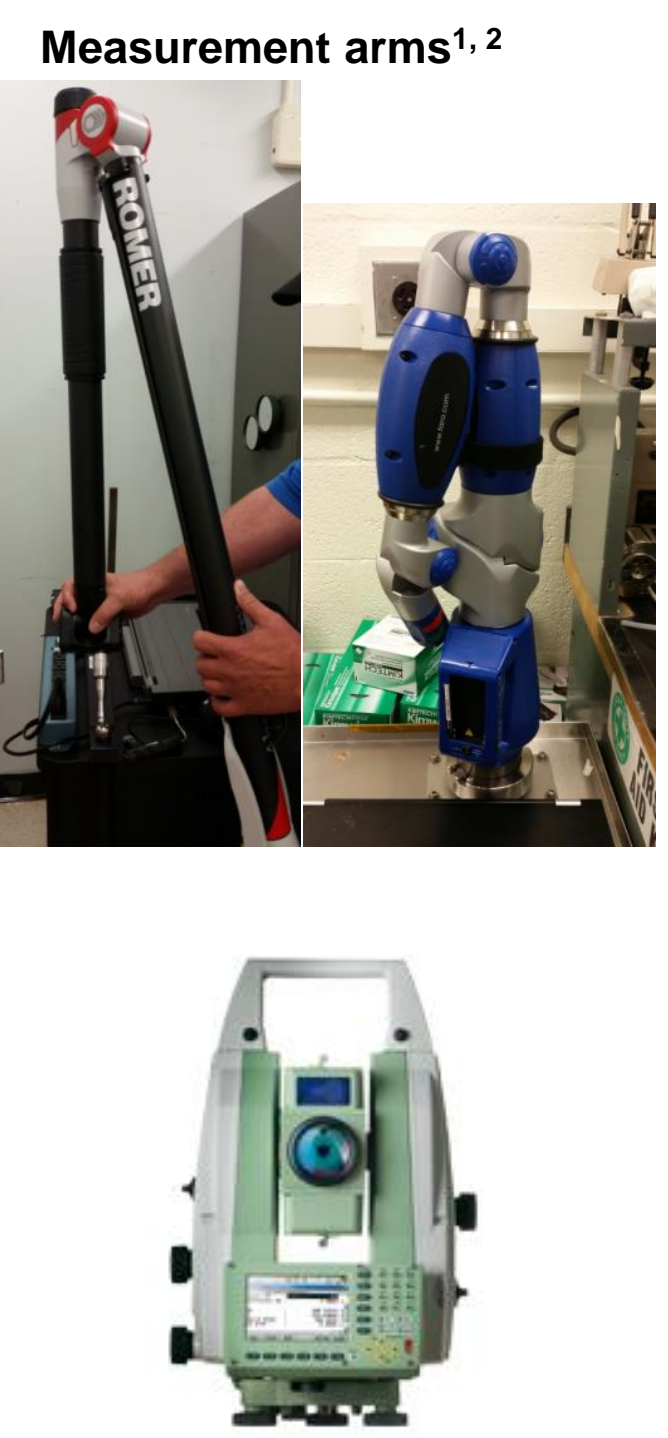

1. www.faro.com

2. www.hexagonmi.com

3. Leica Geosystems AG, Heerbrugg, Switzerland, metrology.leica-geosystems.com 
- Uses precision stages to move measurement probe

- Available in various measurement volumes

- Contact and non-contact measurement probes available

- Test accuracy as low as sub-micron level

- Advantages:

- Highly accurate

- Many probe options including non-contact types

- Disadvantages:

- Limited volume

- Not portable

- "Line of sight" limited

Leitz $^{1}$ Large Volume CMM
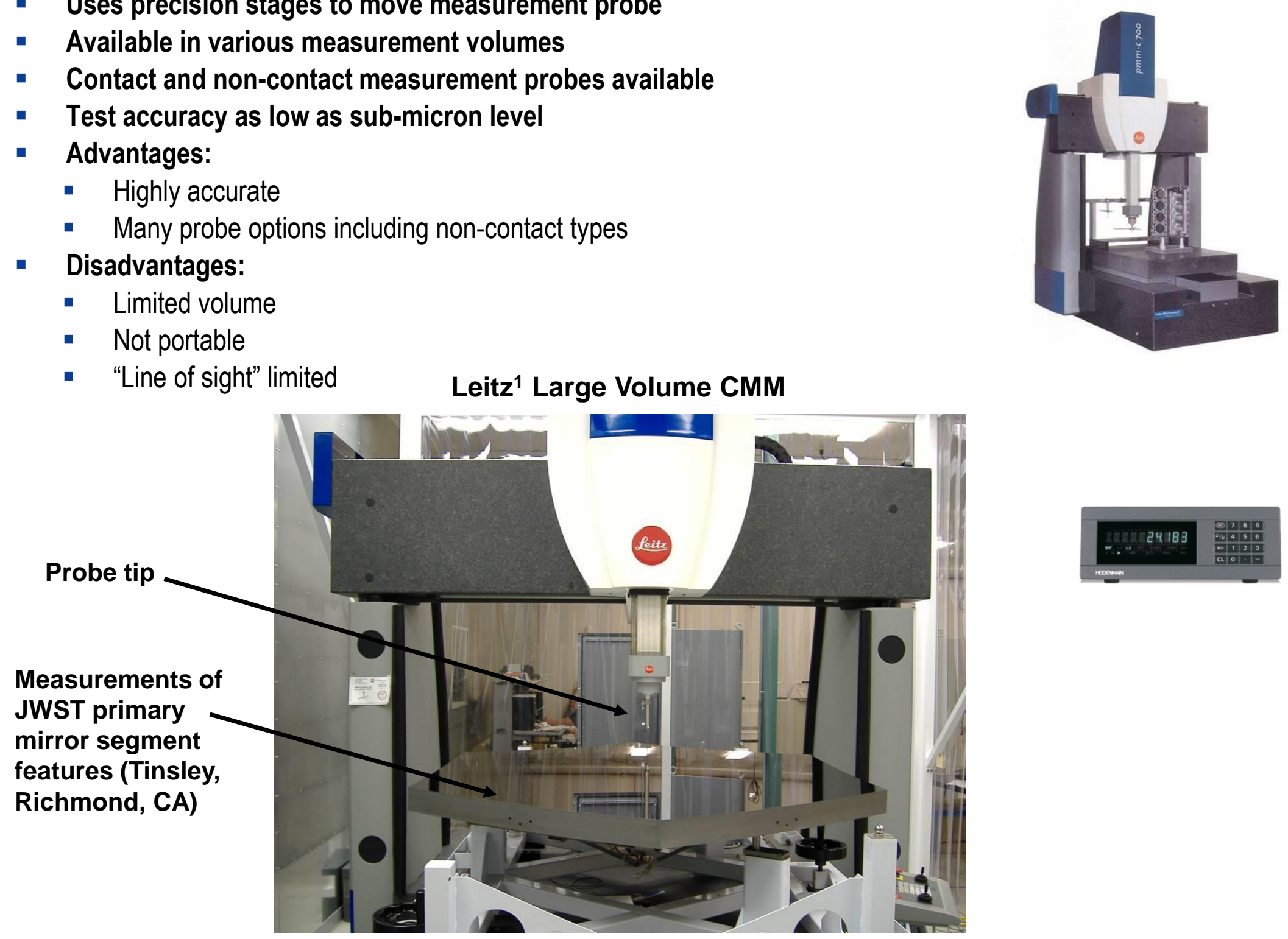

1. www.leitz-metrology.com 
- Provides non-contact measurements in X, Y, Z (3 translational degrees of freedom)

- Microscope coupled with high precision stages with encoders

- Centroid on various targets

- Machined holes

- Metrology targets

- edges

- Accuracy on the order of 20 microns

- Advantages:

- Non-contact measurement

- Highly accurate

- Disadvantages

- Limited measurement volume

- Limited line of sight to target

Micro-vu system measures detector and focal plane fiducials and mechanical interfaces with respect to metrology targets on housing
Micro-Vu ${ }^{1}$ System

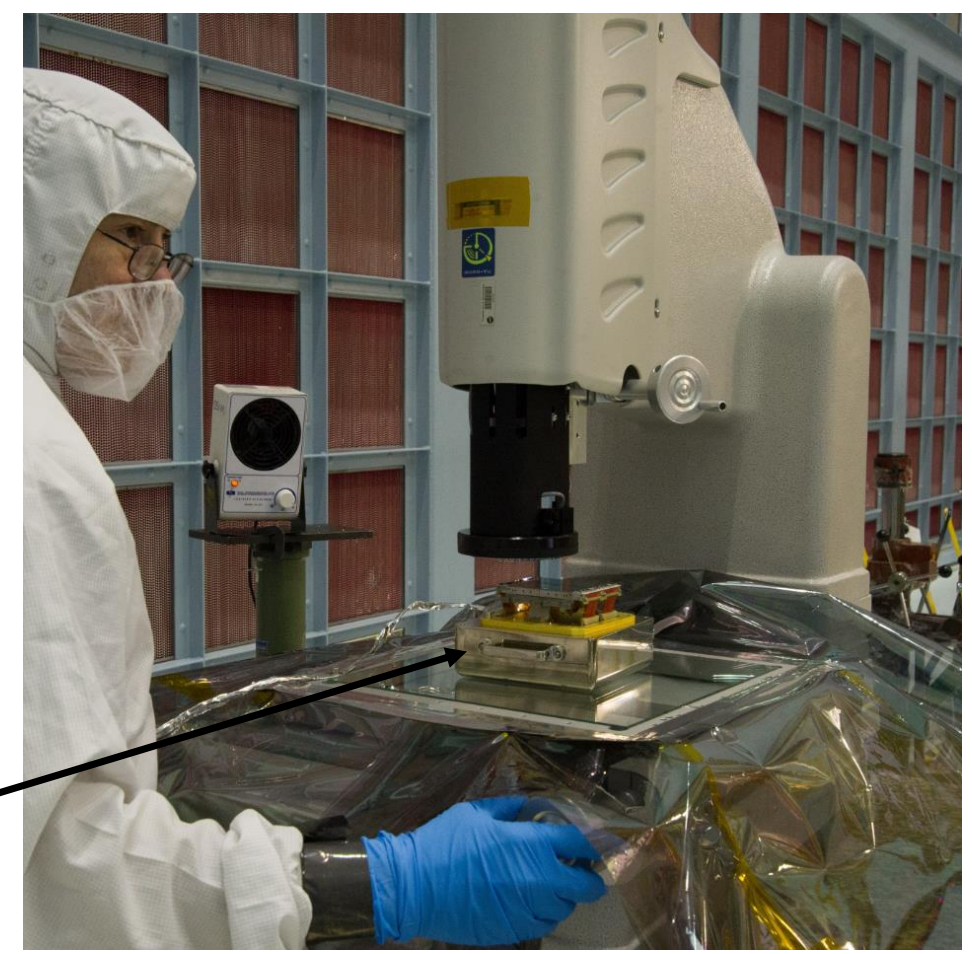




\section{Cathetometer}

- Provides non-contact measurements

- Telescope coupled with high precision stages with encoders

- XY measurement results (2 degrees of freedom) --- single axis version is more commonly available

- Useful for measuring various types of features such as pinholes and detectors

- Accuracy dependent on stage encoders, target distance and human eye

- Advantages:

- Virtually unlimited target types

- Disadvantages:

- Can suffer from cosine error if not aligned well

- Longer setup time. May require theodolites

- Not portable

- Manual data collection

- Measurement area limited to stage travel

Theodolite used to align the cathetometer measurement plane to the target plane via optical cubes

Cathetometer setup to measure pinholes, retroreflectors and flat mirror center locations on JWST ground support equipment 
- Photogrammetry (PG) is used to measure target positions

- Uses specialized cameras which can be mounted or hand-held

- Targets measured from many vantage points to determine location via triangulation; VSTARS ${ }^{1}$

- Its target is a retro-reflective surface with a precision mask

- Scale bars or references are required around the test object

- Some PG targets can be interchanged with TBs or SMRs

- Uncertainty can be as low as $\sim 0.015 \mathrm{~mm}$ (1-sigma; highly dependent on test configuration)

- Advantages:

- Insensitive to instabilities like vibration

- Highly flexible non-contact system

- Large volume

- Can be used under extreme conditions with proper setup

- Disadvantages:

- Limited to measure PG targets only

- Not able to measure surfaces directly without special software

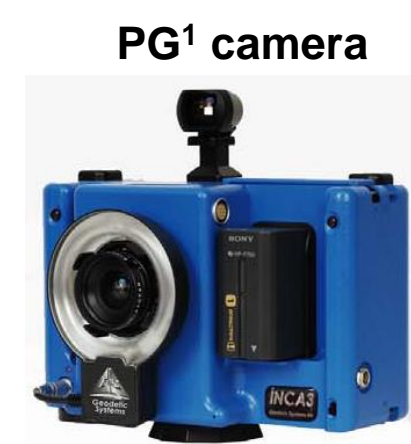

PG target

1. Geodetic Systems Inc., Melbourne, Florida

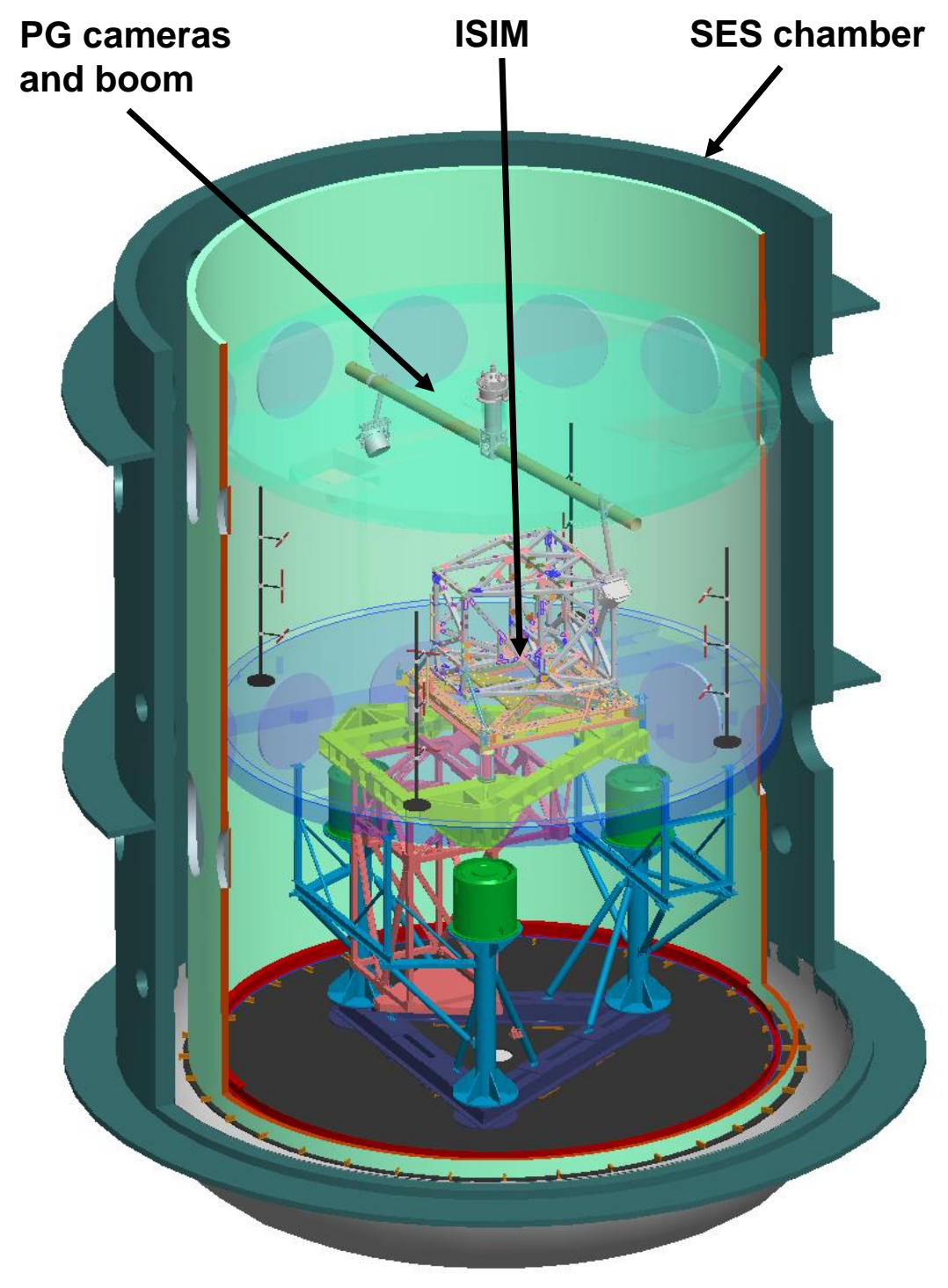


- Produces a spherical wavefront through the system under test

- Images the focal point of the return

- Used to locate centers of curvature or focal point of optical surfaces

- Uses an interchangeble microscope objective at the output of the PSM

- Spheres such as TBs or the back surface of an SMR can be used for alignment allowing easy measurement by LR/LT systems for metrology in the coordinate system

- Can be used as a non-contact CMM probe

- Can also be used as a portable imaging microscope that can be moved to any size work piece

- Can be used as an electronic autocollimator by removing the microscope objective

- Advantages:

- Very flexible and easy to align to optic or system

- Small, portable easy to mount

- Forgiving in angle

- Insensitive to vibration

- Disadvantages

- Needs to be coupled with measurement instrument to get into a CS

- Application is highly setup dependent

TB aligned to PSM focus

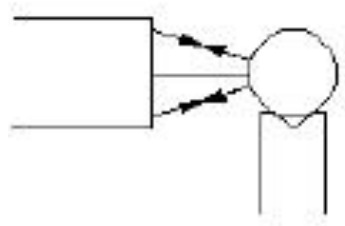

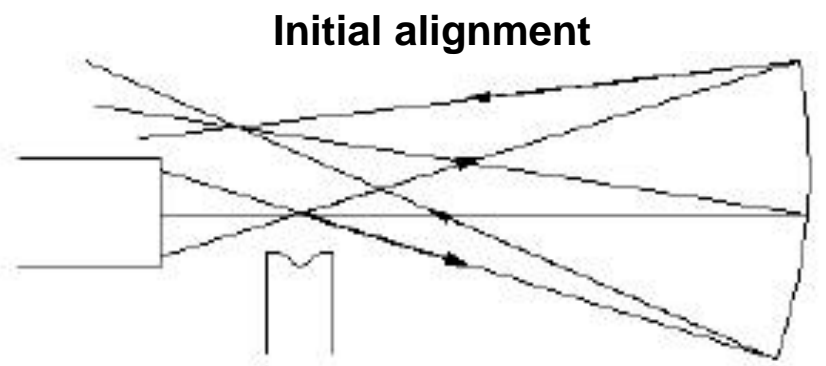

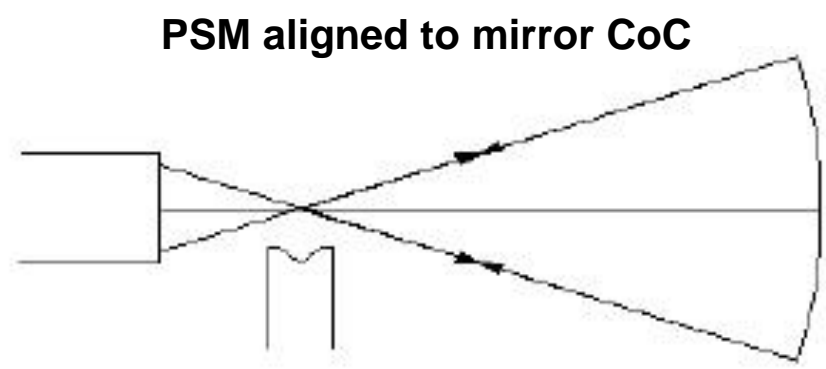

1. Parks \& Borden, "Using the Point Source Microscope (PSM) to find conjugates of parabolic and elliptical off-axis mirrors," Proc. SPIE 8131 (2011)

2. Parks, Robert E. "Autostigmatic microscope and how it works." Applied optics 54.6 (2015): 1436-1438. 
- Theodolites are used to measure angles via auto-collimation and targets via triangulation

- Operated manually

- Angular data is analyzed with GSFC-developed routines (linear algebra)

- Translational data reduced with commercial packages

- Autocollimation: Target is a specular flat mirror (e.g., cube)

- Uncertainty $\sim 2$ arc-sec (1-sigma) for a single measurements, $\sim 30$ arc-sec (1-sigma) for a collection of measurements

- Can be used to measure position via theodolite triangulation

- Typical triangulation targets can be scribe, cross hair or specular tooling ball

- Advantages:

- Large angular measurement range

- Small and portable

- High error when observing at high elevation

- Disadvantages:

- Tough to get into CS

- Theodolite triangulation is challenging

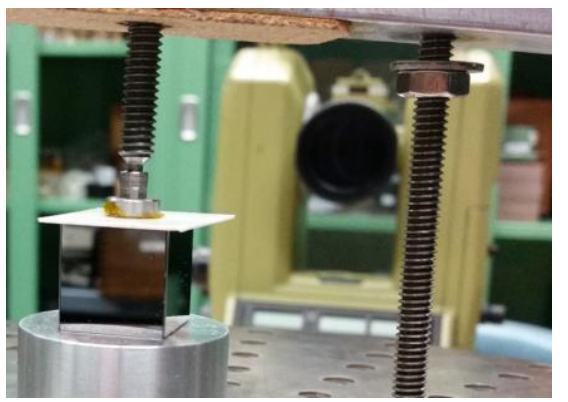

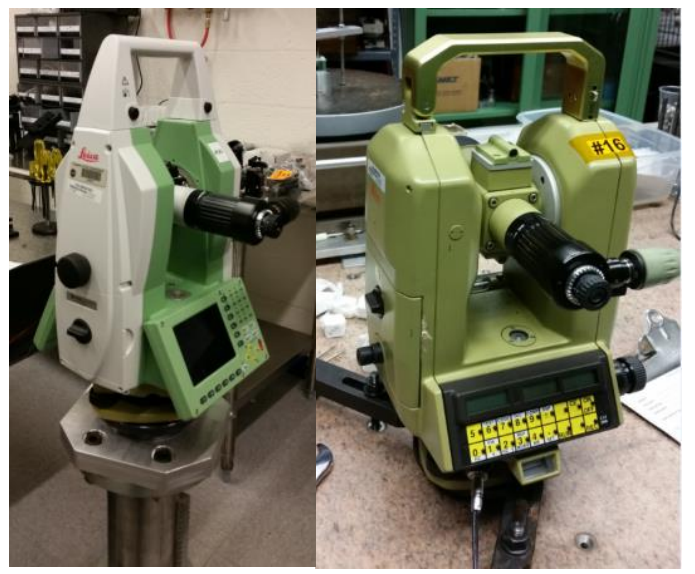

Theodolites

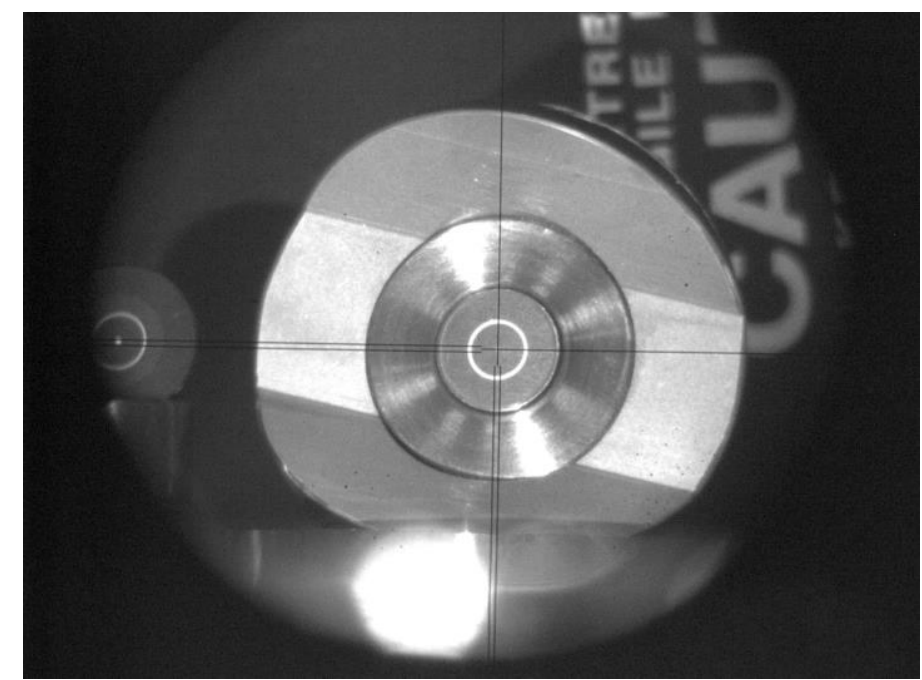

Dot target installed to metrology nest

Optical aliqnment cube 
Autocollimators

- Used to define fixed line of sight (LOS)

- Precise angle measurement

- Resolution on the order of 0.01 arcseconds

- Small angle range on the order of arcminutes

Alignment telescope

- Used to define fixed LOS

- Some equipped with PIP generator for aligning optics

- Some equipped with autocollimators

- Allow vertical and horizontal distance measurement

- Resolution on the order of $0.0025 \mathrm{~mm}$

- Range limited to a few millimeters

Tilt level/Jig transit

- Tilt level used to define horizontal plane

- Measurement range from plane is $2.5 \mathrm{~mm}$

- Measurement increment is $0.0025 \mathrm{~mm}$

- Jig transit measures vertical planes

- Jig transit has cross telescope to measure perpendicular LOS Optical plummet

- Two small autocollimating telescopes pointed up and down

- Used for vertical systems

- Useful with aligning elements parallel with gravity
Alignment Telescope
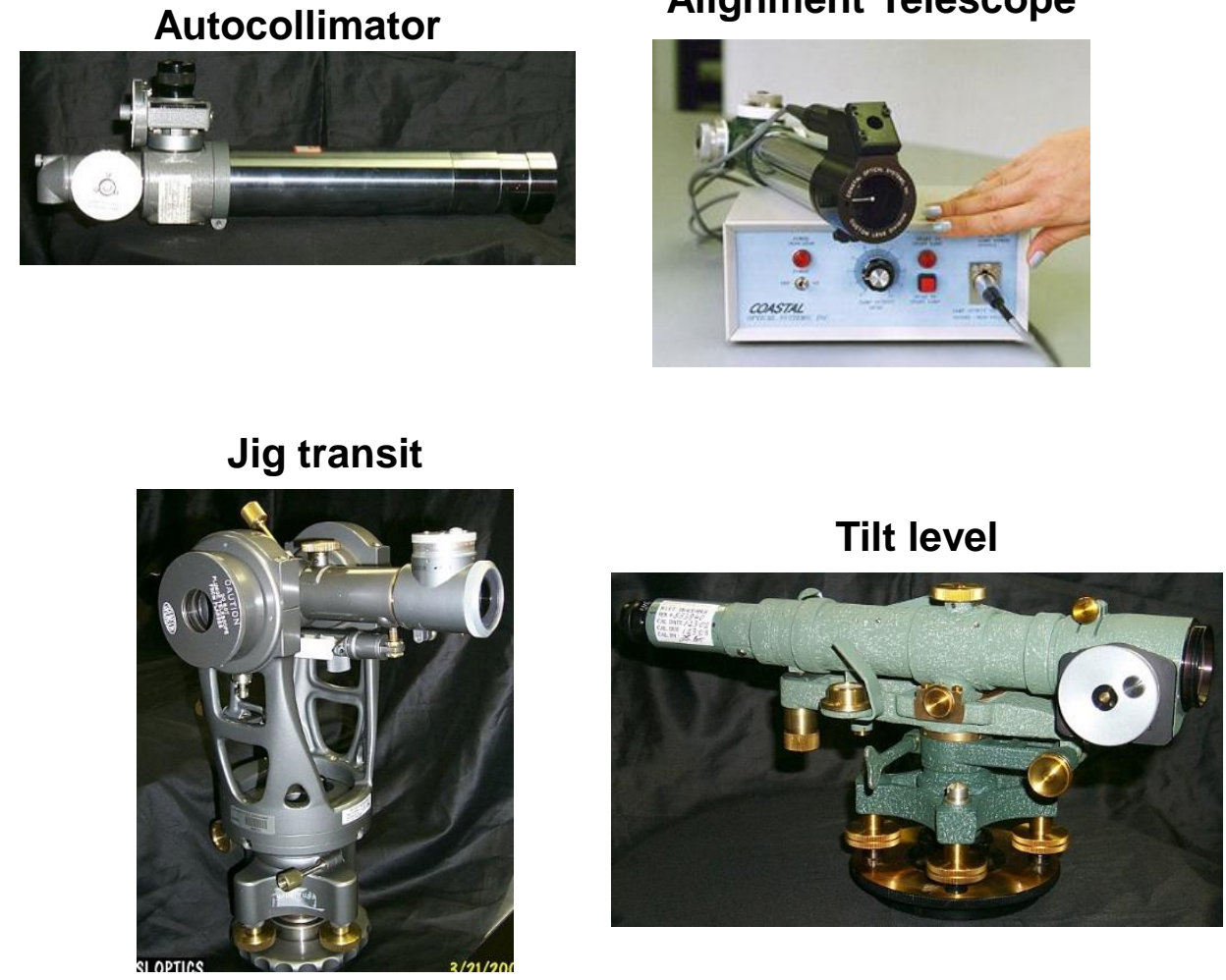

Optical plummet

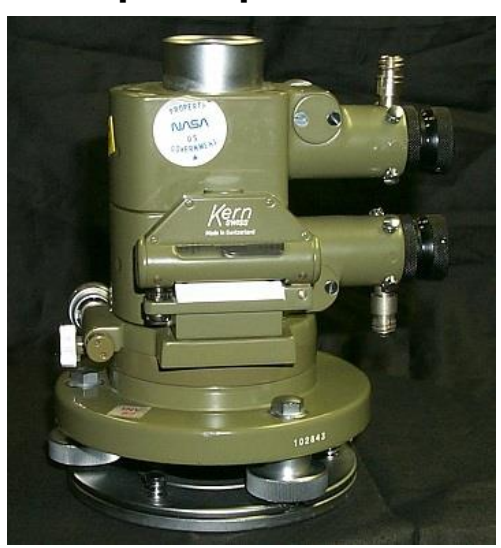


- Measurement data processing

- Statistical tools 
- GSFC uses Spatial Analyzer ${ }^{1}$ (SA) software to collect and process data from LT, LR, Total station, Arms and some theodolite data --- other, similar software packages exist from other vendors to address the same need

- Process scan data to fit to shapes and CAD models

- SA combines results from multiple instruments using USMN2 function

- Can be used to import data from other measurement instruments (Cathetometer, CMM, Arms, etc.)

- Other software packages available with similar capabilities

- CMMs and measurement arms data is typical collected using manufacturers software

- Theodolite angular data is processed manually using NASA GSFC created macros for Microsoft Excel ${ }^{3,4}$
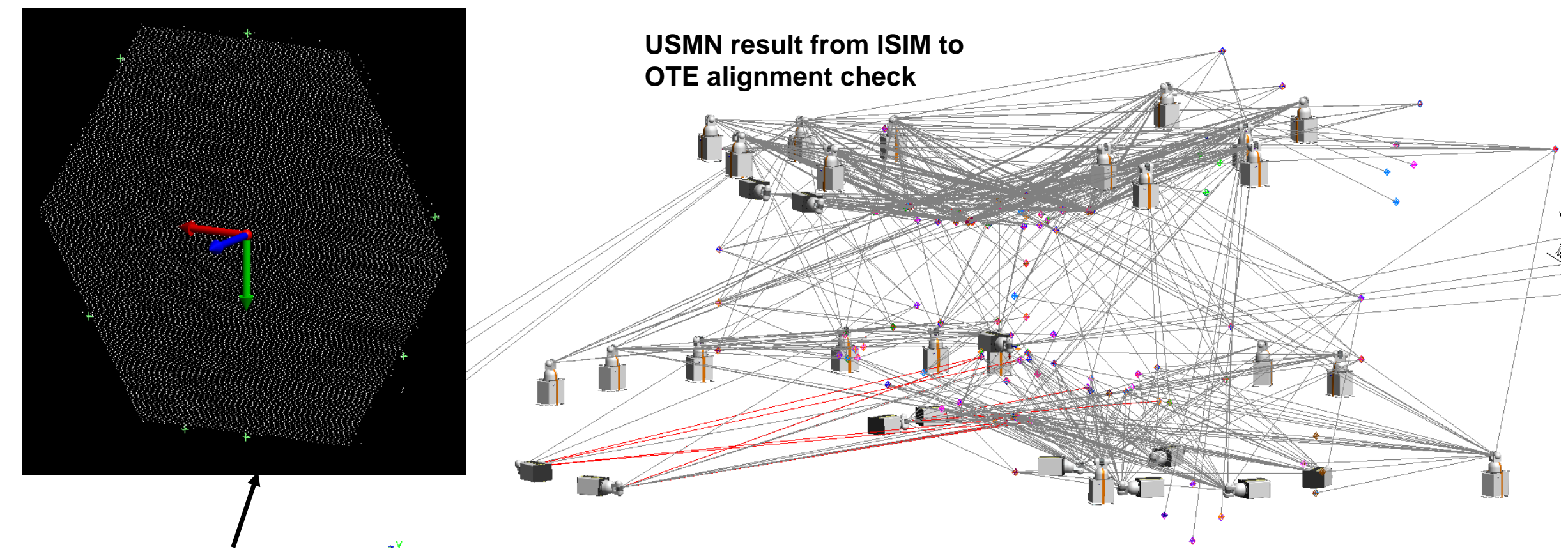

Processed LR scan data in SA for one of the JWST primary mirror segments

1. New River Kinematics, Inc., Williamsburg, Va.

2. S. Sandwith \& R. Predmore, "Real-time 5-micron Uncertainty with Laser Tracking Interferometer Systems using Weighted Trilateration," New River Kinematics, Inc. Williamsburg, Va.

3. C. Aviado, J. Gill, K. Redman, and R. Ohl, Methods for correlating autocollimation of theodolites and coordinate metrology in spacecraft systems, Proc. SPIE 6273, 62733H, 2006

4. W. Eichhorn, "Optical alignment measurements at Goddard Space Flight Center," Applied Optics 21, 3891, 1982 
- To calculate measurement uncertainties measurements are typically taken in 3 sets (minimum; often 5 or more) and from multiple stations to help reduce sources of systematic error

- Microsoft Excel can be used to calculate Student's- $t^{1}$ uncertainties for low sample statistics --- other ways exist to handle small number statistics

- Custom routines can be created using MATLAB' ${ }^{2}, \mathrm{IDL}^{3}$, and spreadsheets to calculate uncertainties

- JWST MCTU4 uncertainty propagation routine

- Used to propagate uncertainties throughout JWST databases and to estimate transformation uncertainties

- SA USMN 5 function calculates measurement uncertainties based on the instruments used

- Other metrology software packages available with statistical functions

- Custom Excel macros have been created at GSFC to calculate statistics, including student's-t, for angular theodolite data

USMN resulting point uncertainty clouds (exaggerated) from SA

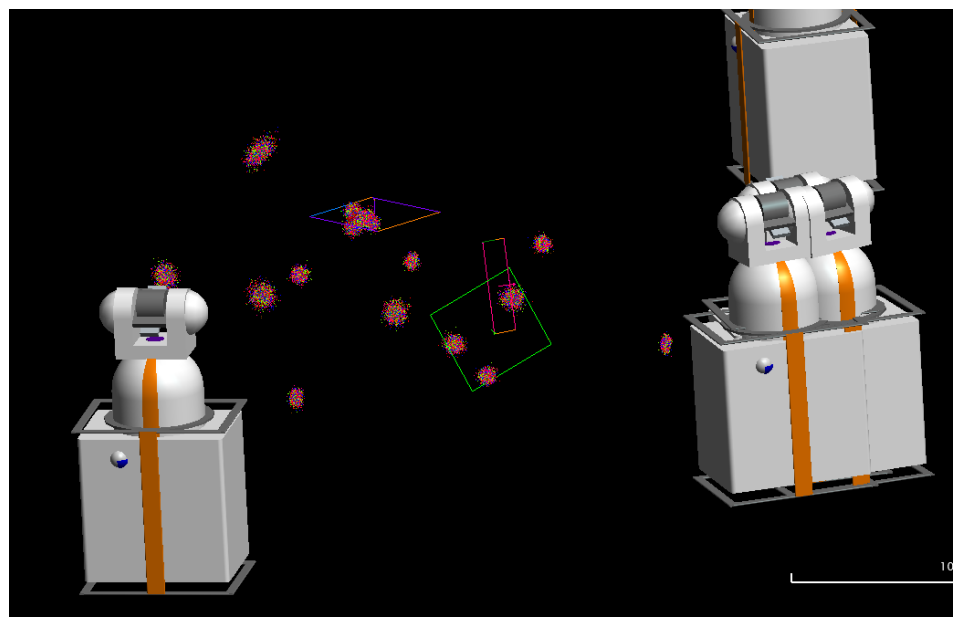

1. J. Hayden et al., "Measurements and Analysis used for the Determination of the James Webb Space Telescope Integrated Science Instrument Module Vehicle Coordinate System," Coordinate Metrology Society Conference, July 2010.

2. www.mathworks.com

3. www.harrisgeospatial.com

4. J. Hayden, M. Khreishi, T. Hadjimichael, and R. Ohl, Monte Carlo Method for Uncertainty Propagation in JWST Metrology Databases, Coordinate Metrology Systems Conference, 2014.

5. S. Sandwith \& R. Predmore, "Real-time 5-micron Uncertainty with Laser Tracking Interferometer Systems using Weighted Trilateration," New River Kinematics, Inc. Williamsburg, Va 


\section{(190) Applications}

- How are these tools used to measure optical surfaces and systems?

- That's what we really care about!

- Some recent examples:

- James Webb Space Telescope-ISIM to OTE

- OSIRIS-Rex/OVIRS
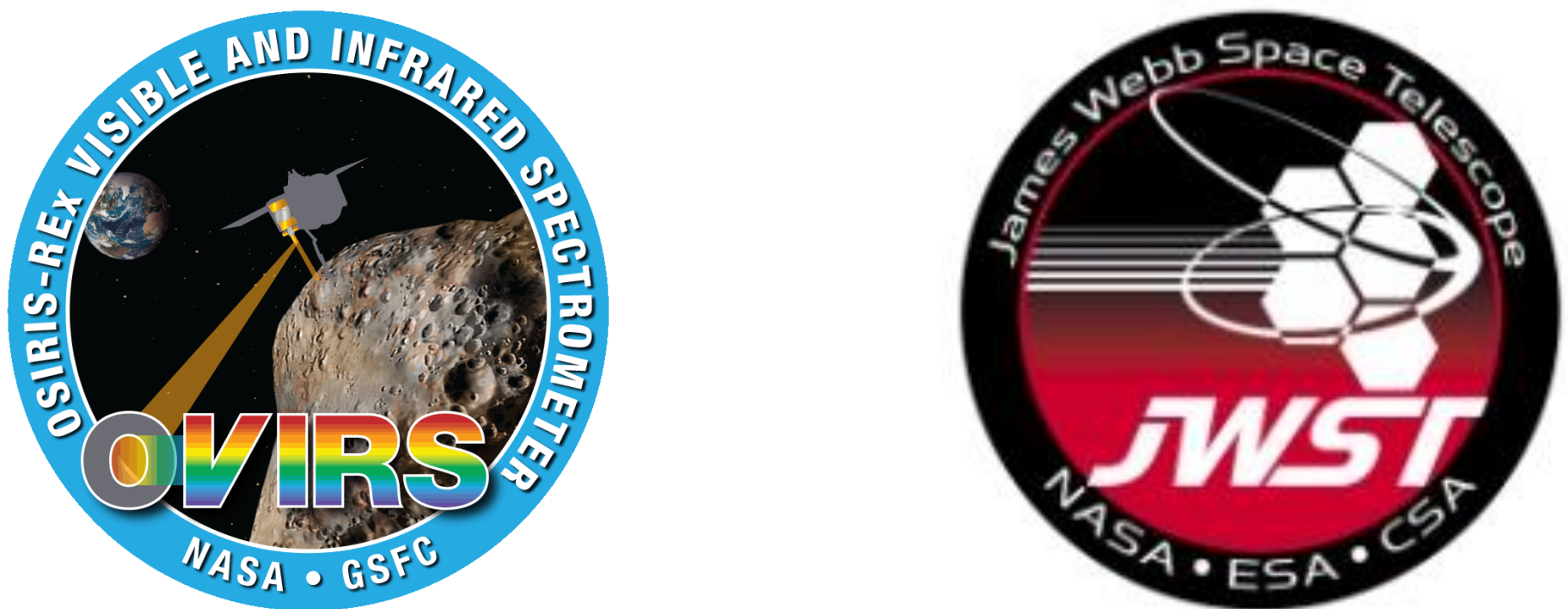


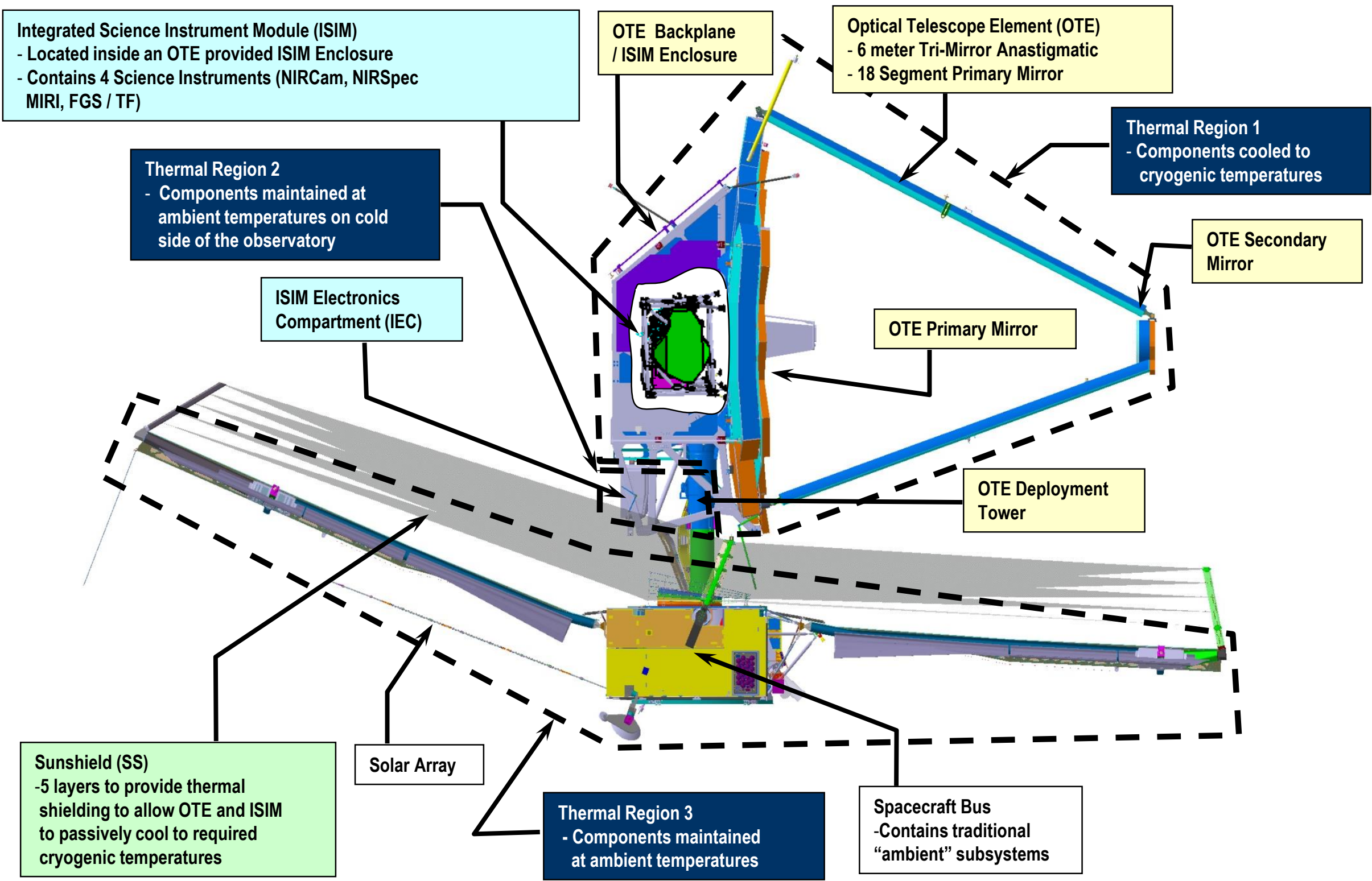


references with offsets and evaluation via optical analysis

- In May 2016 the Integrate Science Instrument Module (ISIM) module was integrated to the Primary Mirror Backplane Support Structure (PMBSS)

- Prior to integration extensive tolerance analyses were performed to understand 6 DOF alignment sensitivities

- ISIM alignment sensitivities were investigated as a function of Kinematic Mount (KM) strut lengths and spherical end positions

- Prior to integration a series of measurements were conducted to predict the ISIM pose

- KM strut length

- Spherical seat measurements of ISIM and PMBSS

- Metrology target measured on ISIM and PMBSS in separate tests

- As-built ISIM performance evaluated --- offsets were not necessary

- As-built OTE performance evaluated --- offsets were not necessary

- During installation "Transtrack" was used to ensure hardware safety

- Measurement were conducted post-integration of ISIM to the PMBSS structure

- A network of LR systems measured both structures from multiple stations

- The predictions and final post-alignment measurements checked that ISIM-OTE met alignment requirements with margin at ambient

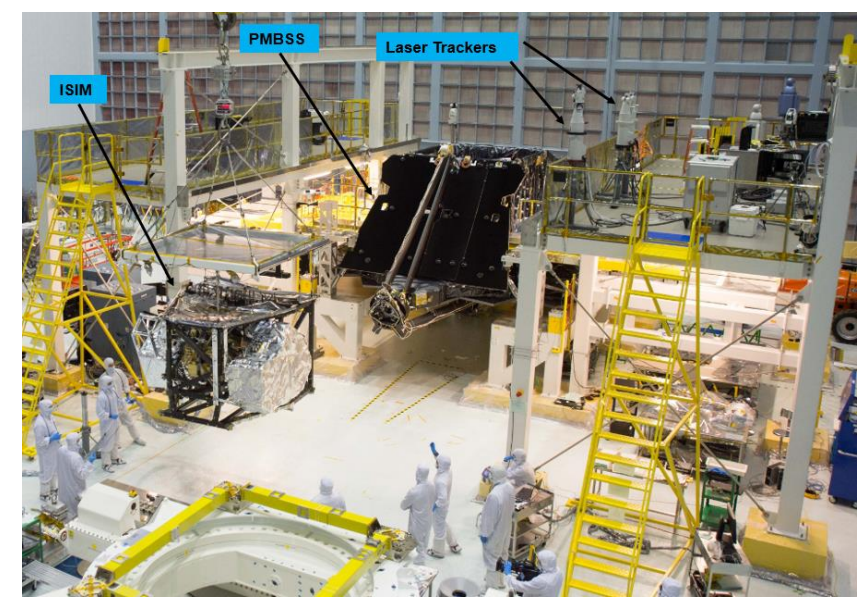

1. Hubler, D., et al. "Using Real-Time, 6D Object Tracking to Assemble Large Aerospace Components." 2000 CMSC Conference Detroit, Michigan. 2000. 
- Multiple LR stations around the PMBSS structure to reduce measurement uncertainties

- USMN result showing all LR stations including shot lines to targets

- Points on the outside represent scale bar checks

- Some stations required the LR to be mounted on its side to allow line of sight to some of the lower targets

Multiple LR stations

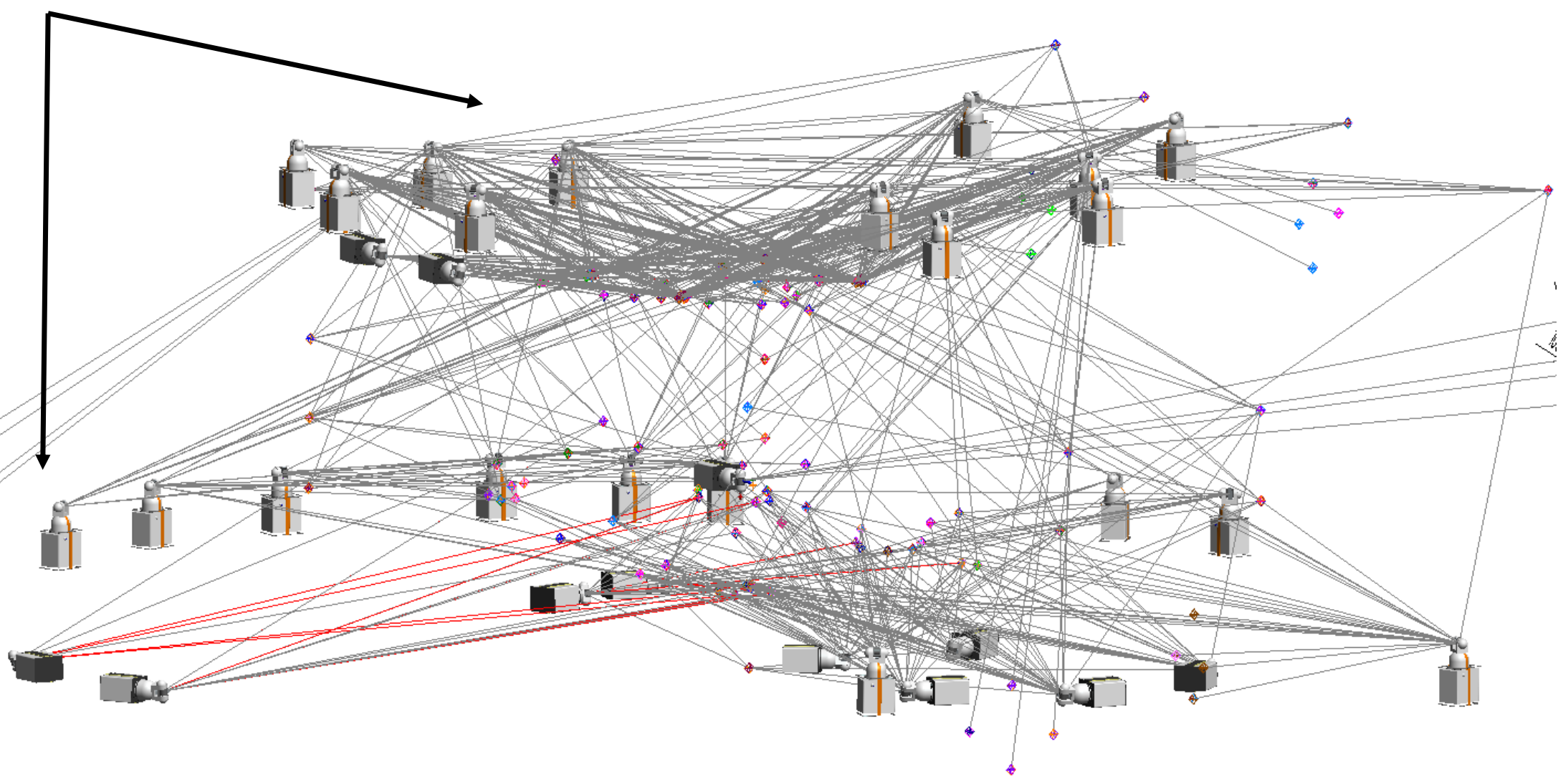




\section{NASA JWST ISIM to PMBSS Alignment Verification}

- LR was used to scan one of the JWST primary mirror segments

- Goal was to:

- Measure the mirror edges to help locate the position of the segment during integration to the backplane

- Measure the front surface of the mirror segment

- Mirror edge measurement was successful to the requirements due to the laser beam size

- Mirror scan of the front surface was successful

LR scan result Multiple LR stations

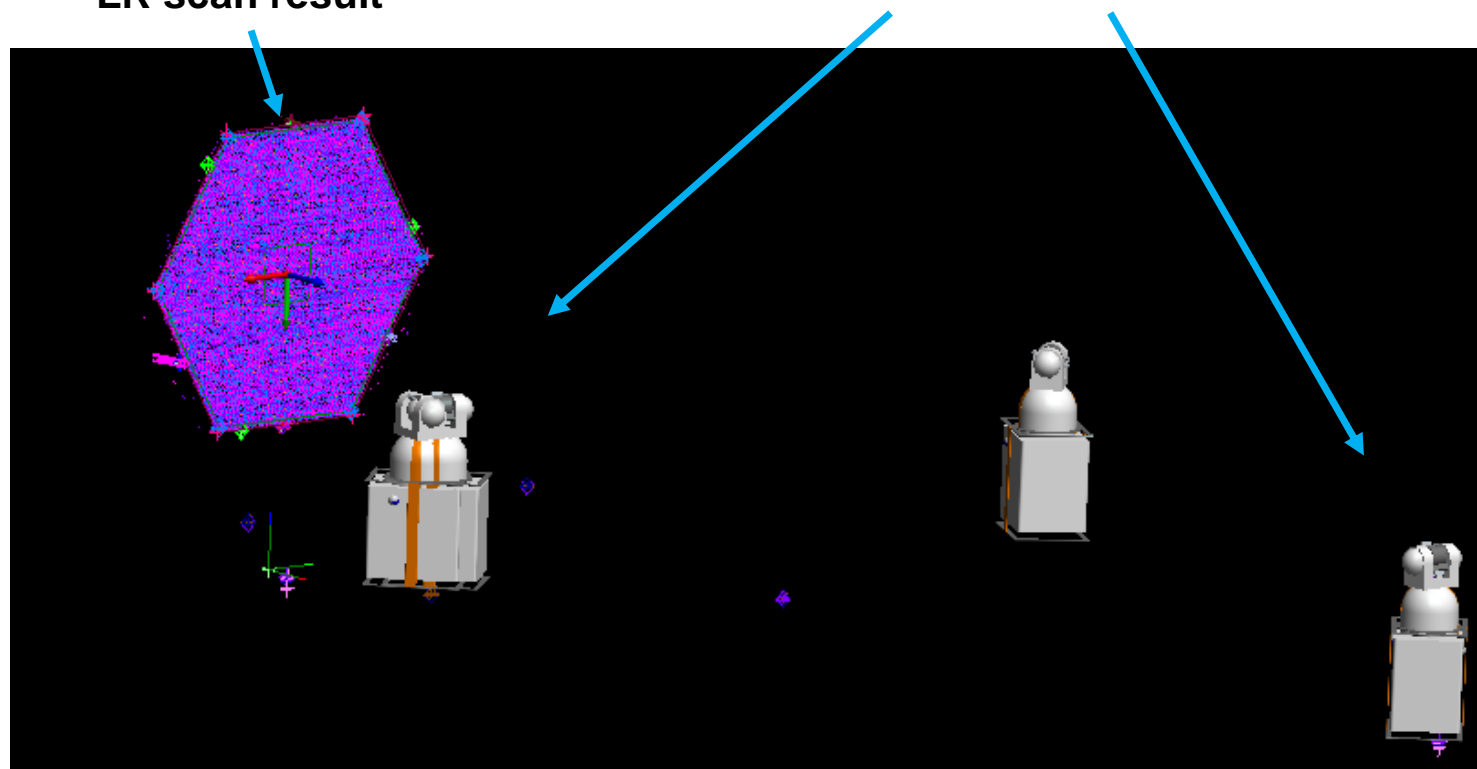


Nate Applications: OVIRS instrument development is an example where metrology is used more directly for optical surface alignment
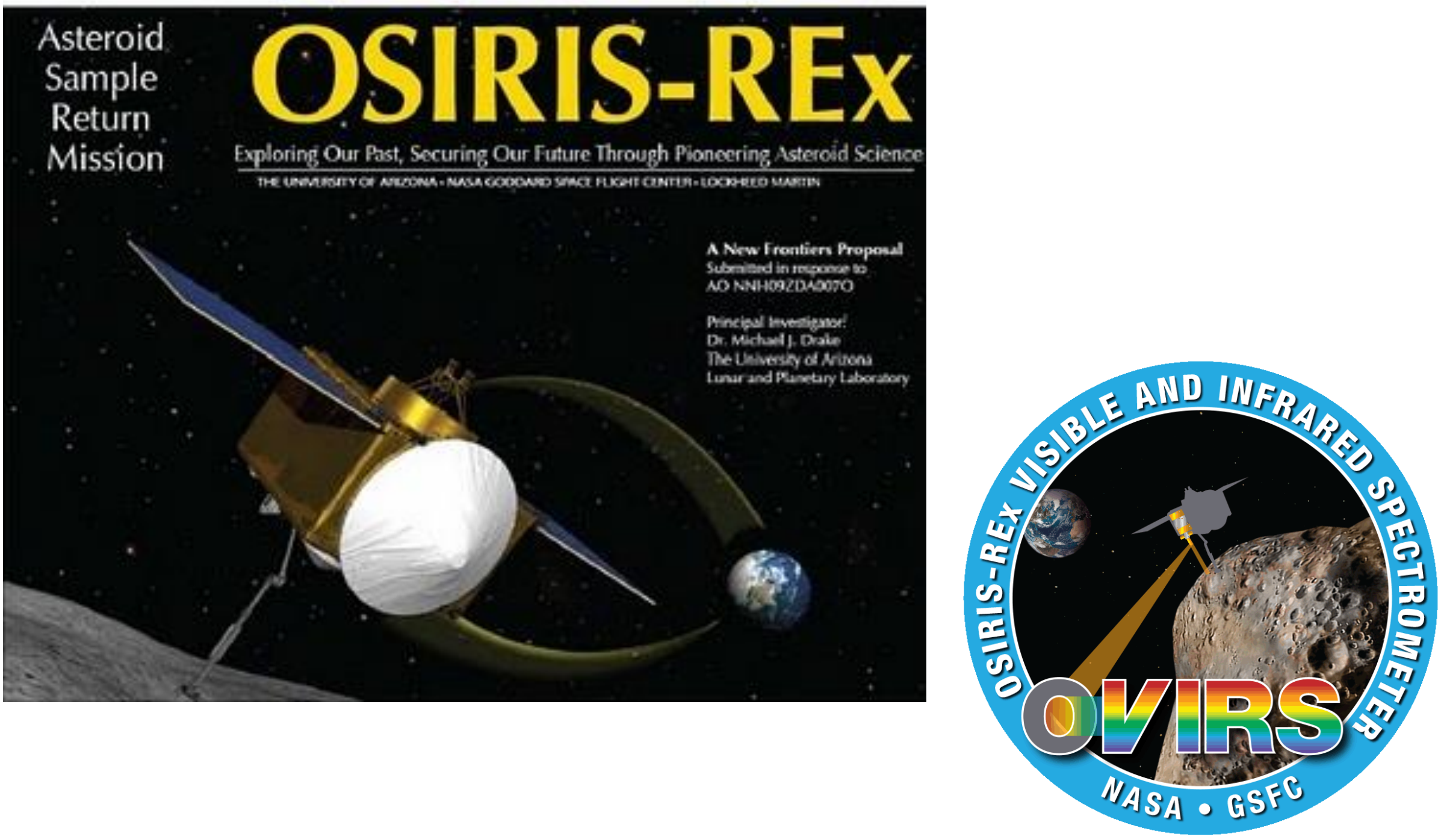


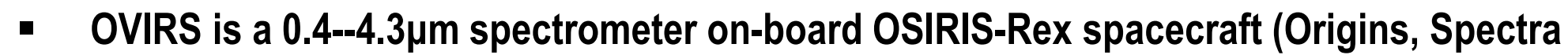
Interpretation, Resource Identification, Security, Regolith Explorer)

- Four key optical performance parameters

- Collimation quality (angle of incidence at filter) $\rightarrow$ impacts resolving power

- Field of view $\rightarrow$ beam footprint on asteroid

- Boresight $\rightarrow$ ensures overlap with other OSIRIS-REx instruments

- Effective throughput $\rightarrow$ impacts signal-to-noise ratio

- Various measurement instruments were used throughout I\&T

- Laser radar

- Laser Trackers

- Theodolites

- Interferometer

- Microscope Optical CMM

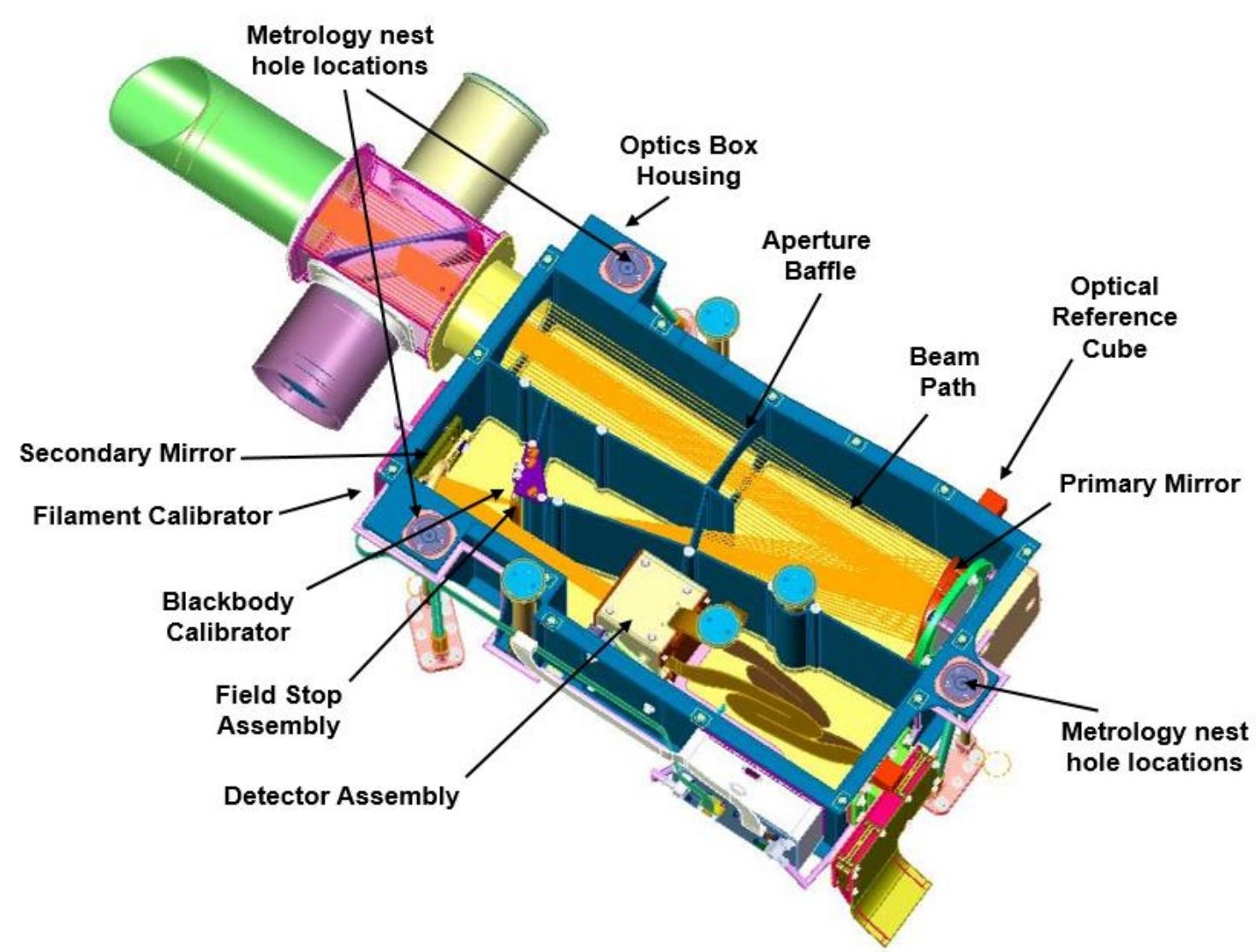


- Component level characterization

- Primary and secondary mirrors (surface error, alignment fiducials)

- Field stop

- Detector assembly

- Characterize optical box/bench ("O-box") to define coordinate system an measure key features, like interfaces to the spacecraft and optical elements

- Install and align components to bench based on fiducials

- Test system

- System wavefront error at ambient

- Optical boresight and ambient (and the cryogenic operating temperature)

- (Science performance at the cryogenic operating temperature)

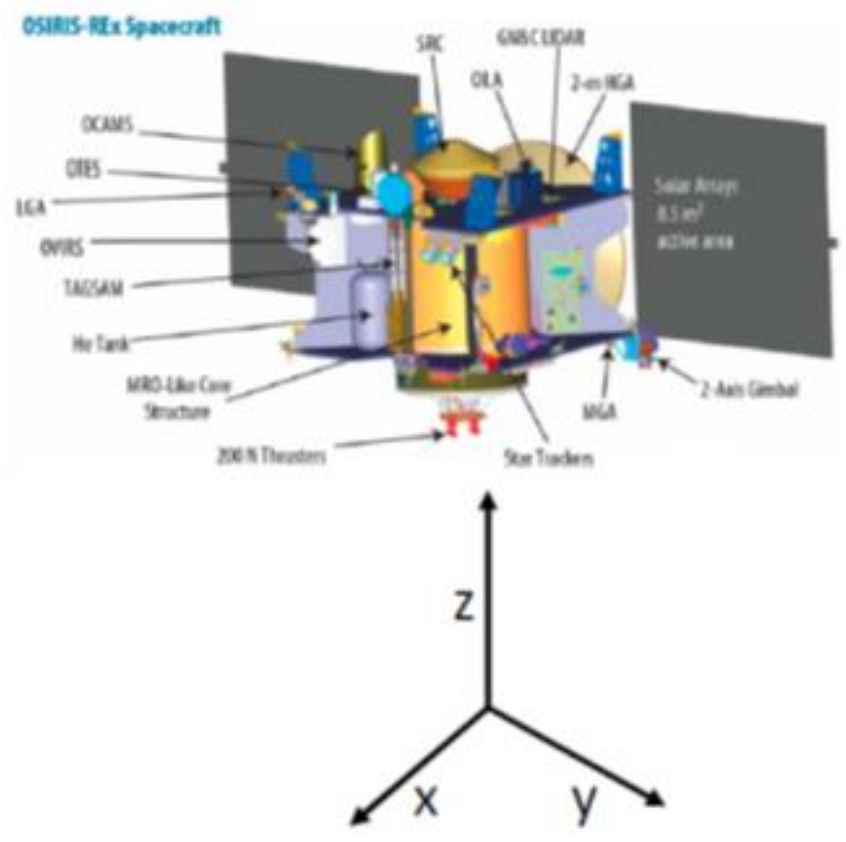



for six degrees of freedom positioning

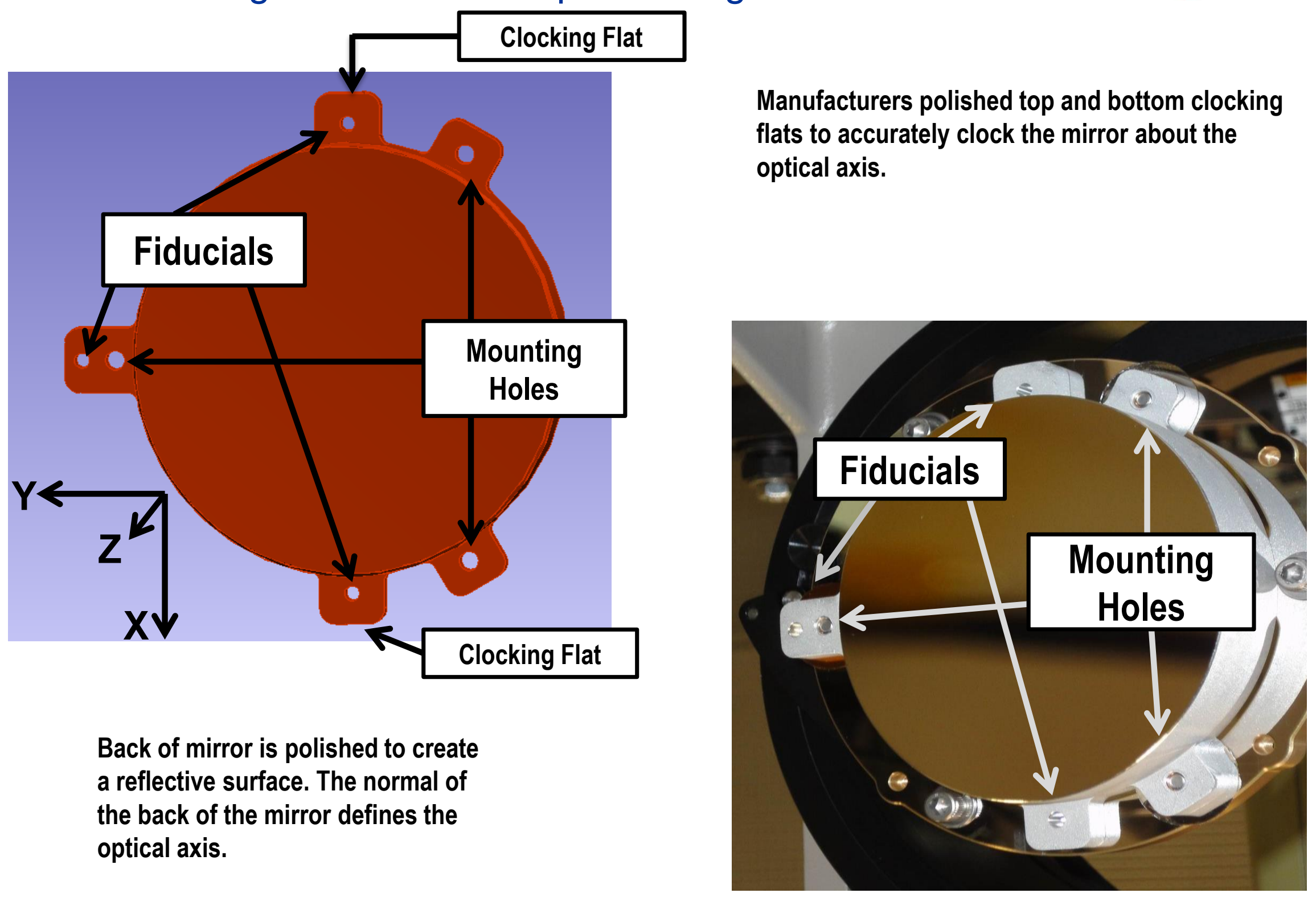



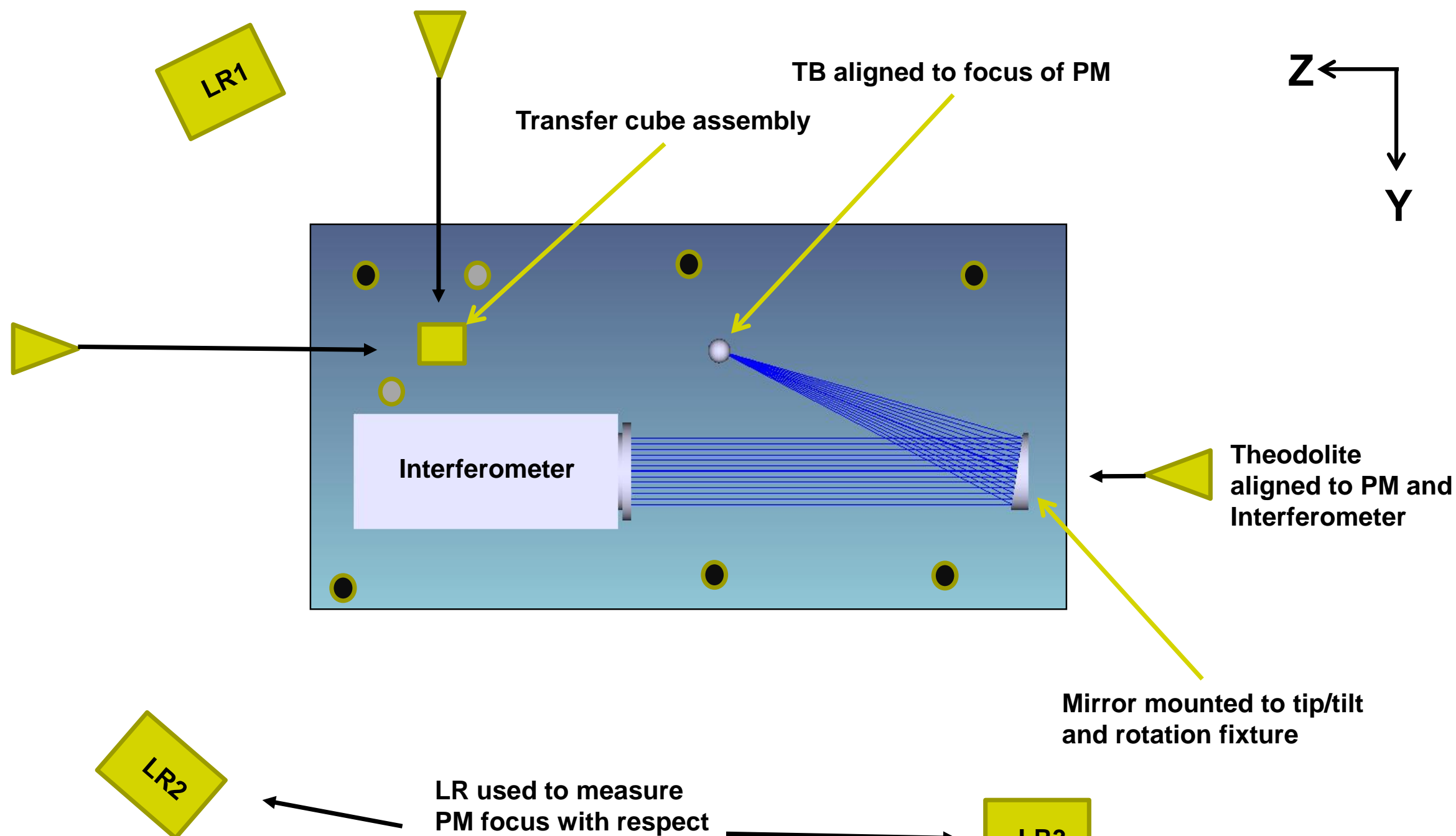

LR used to measure

PM focus with respect

to mirror fiducials

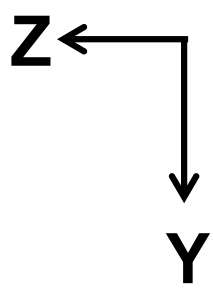

from various stations

Mirror mounted to tip/tilt and rotation fixture 


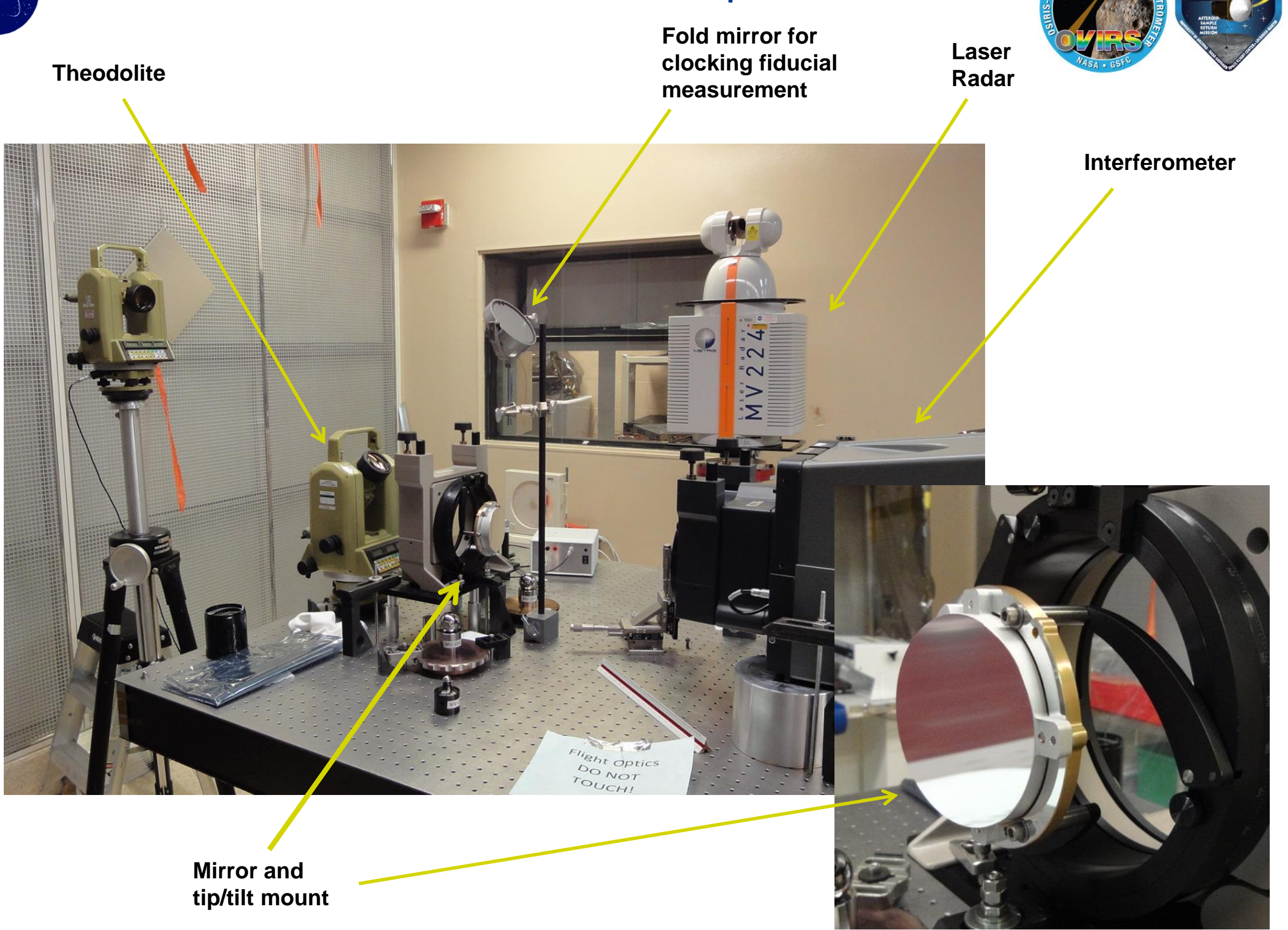




\section{Detector Characterization}

- Detector opening measured with respect to metrology nest targets

- Micro-Vu microscope ("optical CMM") was used to characterize the detector assembly prior to installation

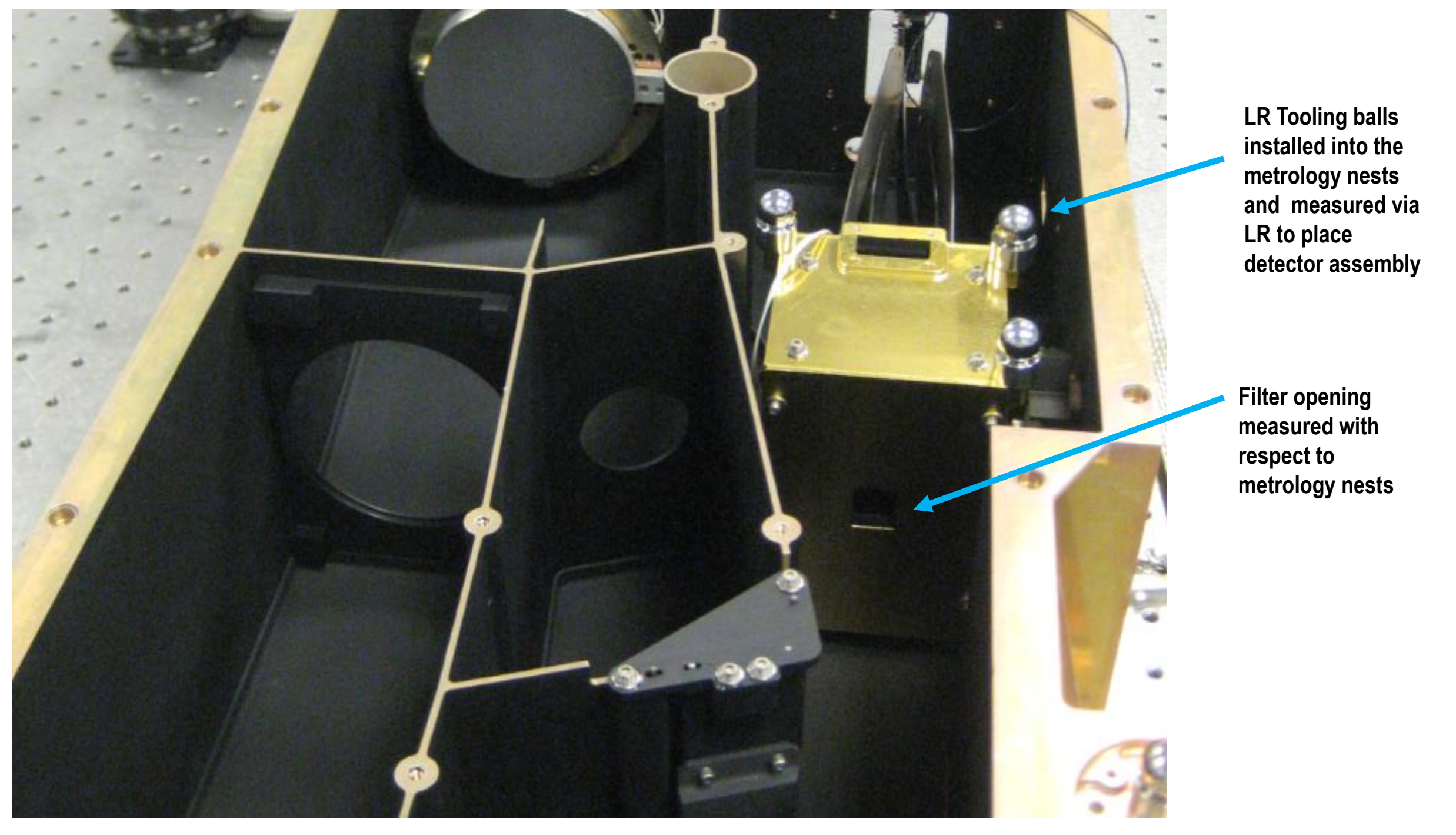




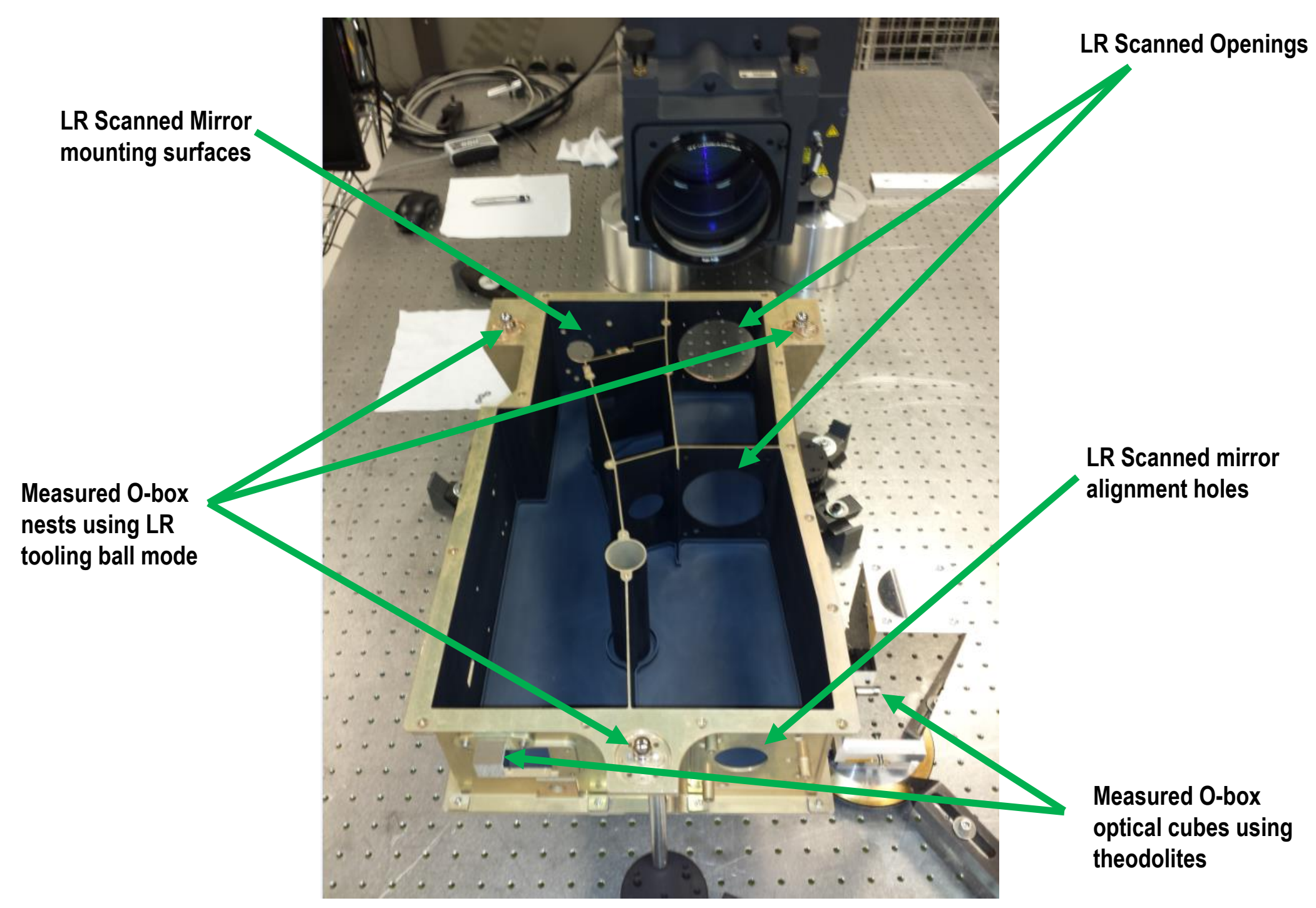


- OB metrology targets define the CS of the optical box

- $O C$ cubes are used as a reference to the boresight

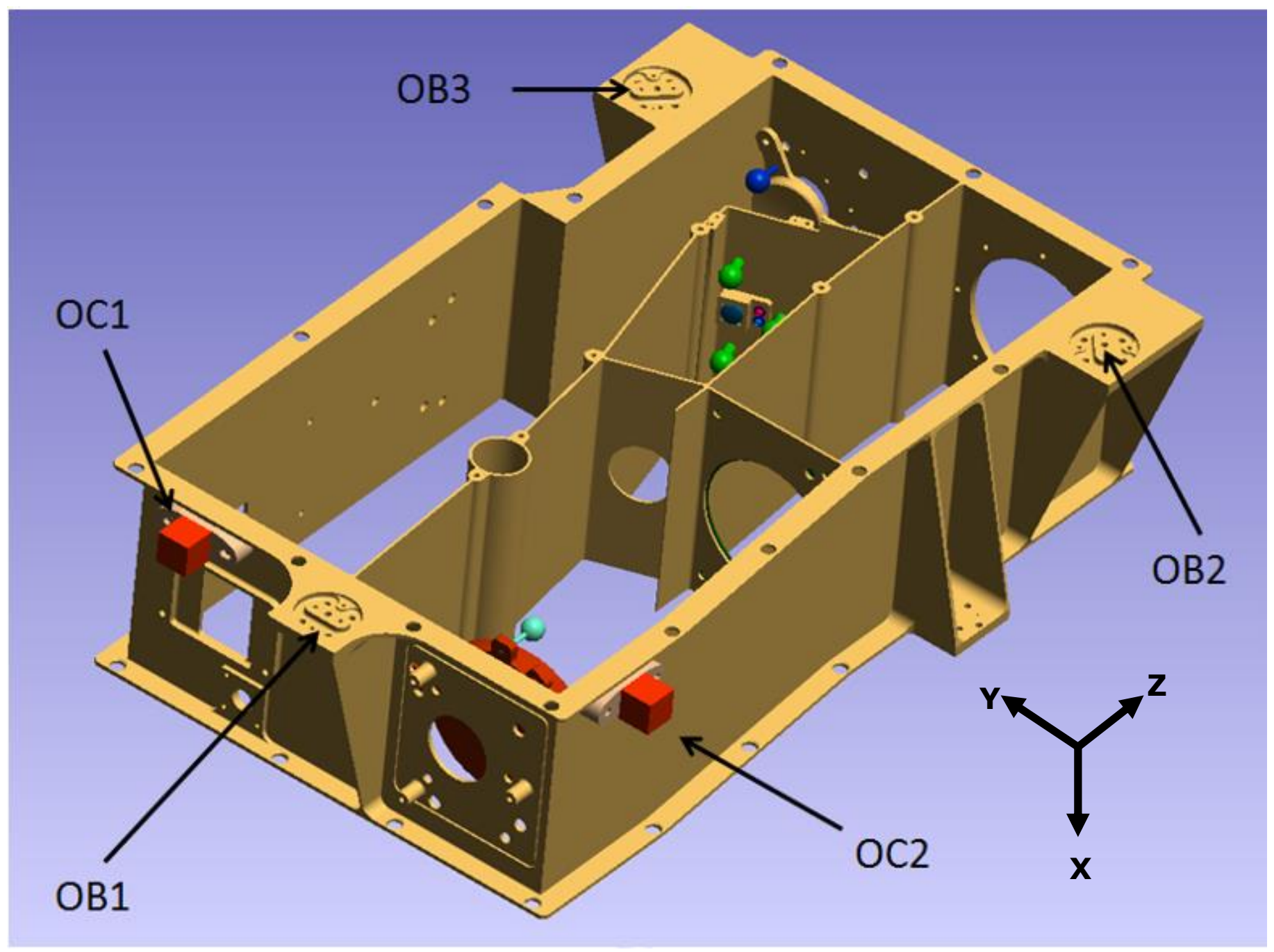




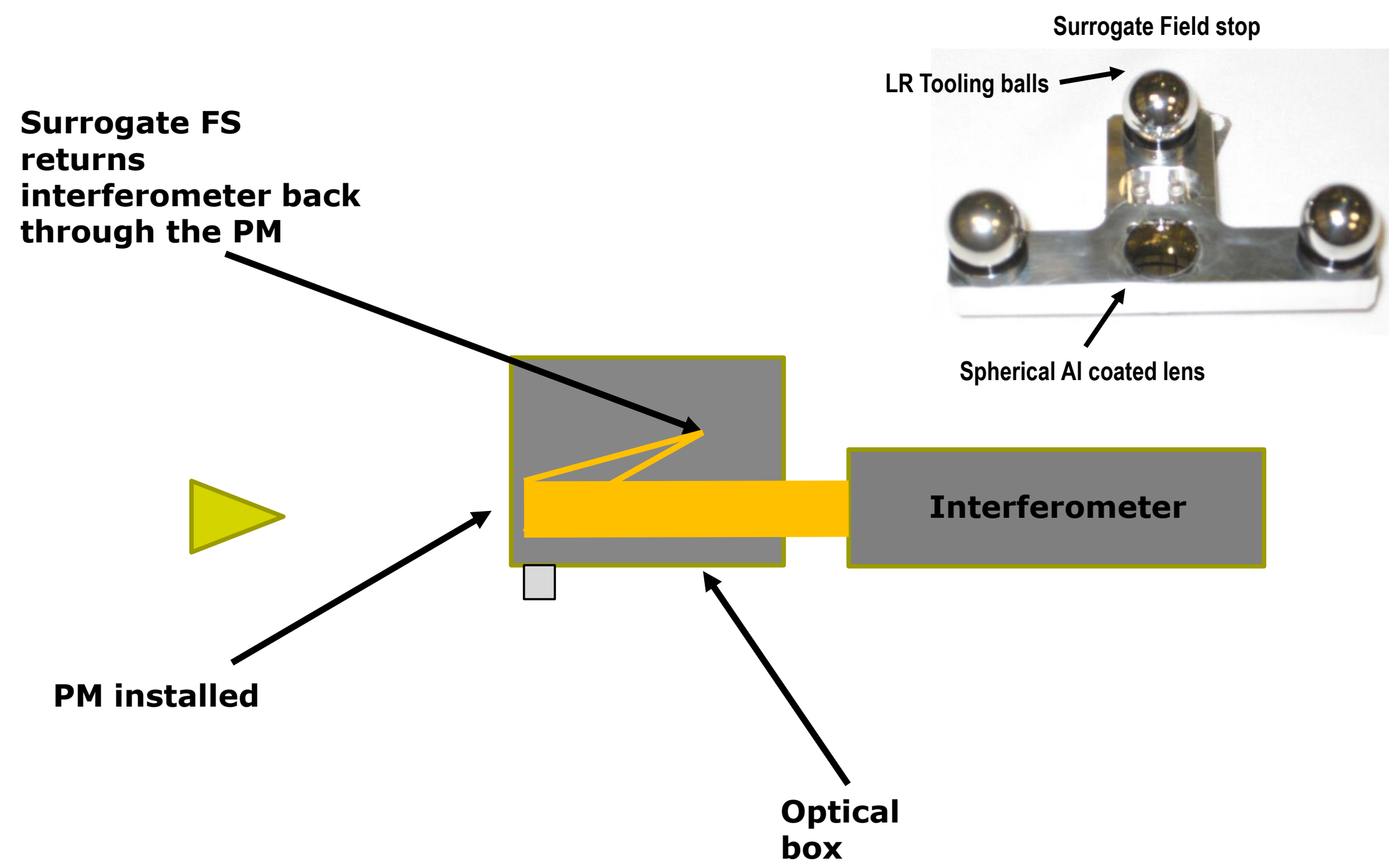


Large Flat mirror returns interferometer beam through the system.
Surrogate FS replaced

with the flight FS
Theodolite setup on the PM rear surface

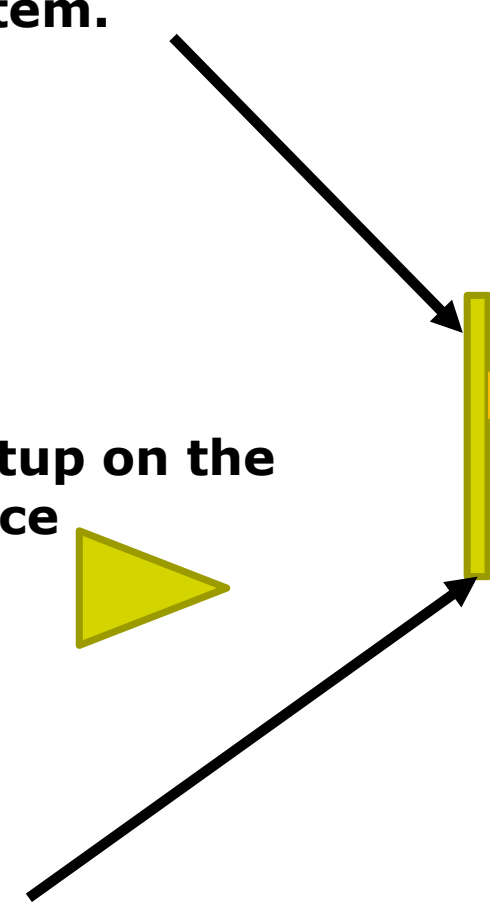

The mirror is oversized such that the interferometer can see a direct return

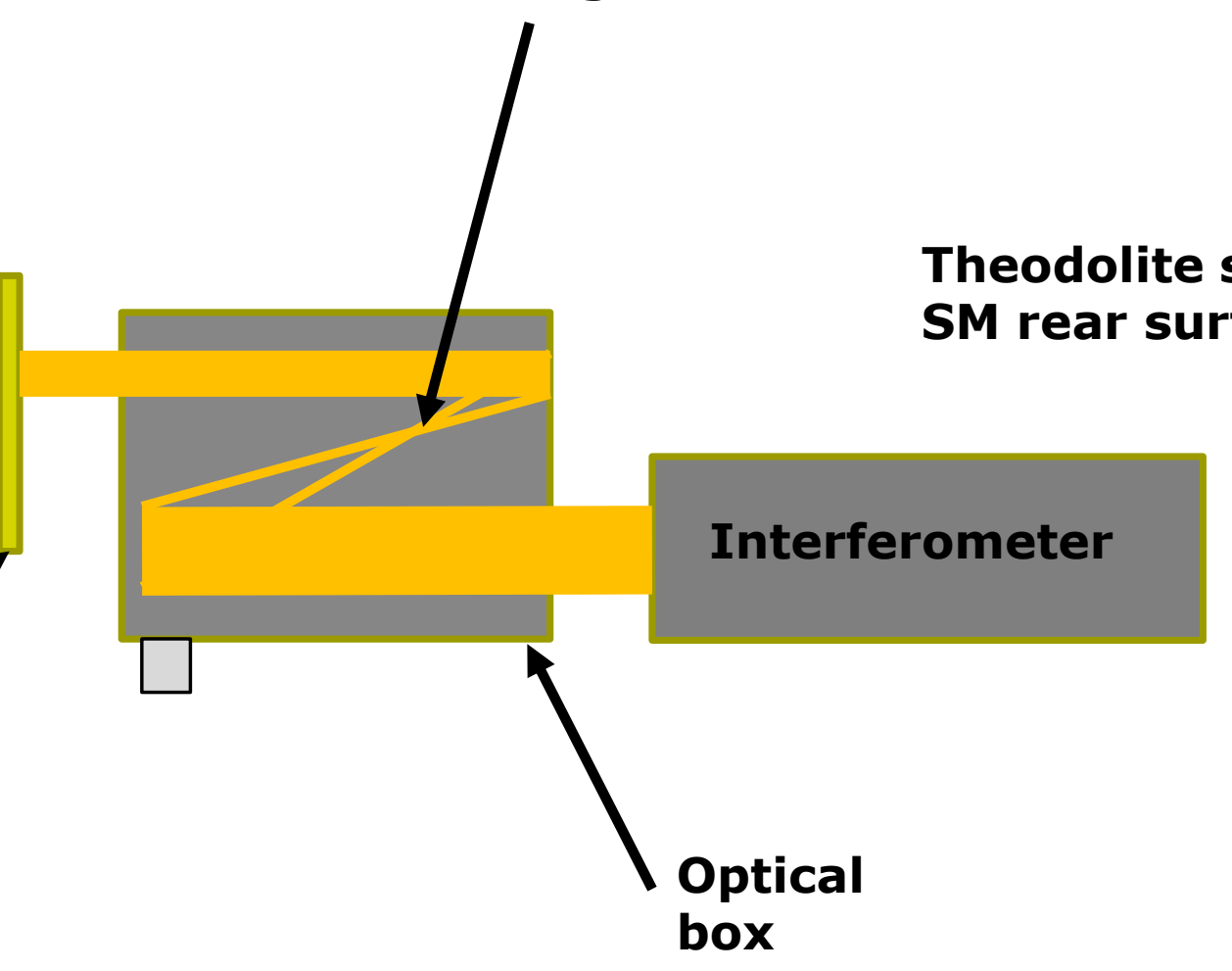

Theodolites measure Obox cube with respect to the interferometer output to determine the ambient boresight 
- Ambient boresight measured using theodolites in interferometer test setup

- Cryogenic boresight measured in thermal vacuum chamber after installation of the detector

- Measurements of OVIRS and optical calibration GSE (optical cubes) were made in the test chamber

- A small laser tracker was used due to space and stability constraints

- A "Direct and Through" technique was also used to measure one of the cube surfaces

- A network of tie points was mounted throughout the chamber to tie in all of the measurements

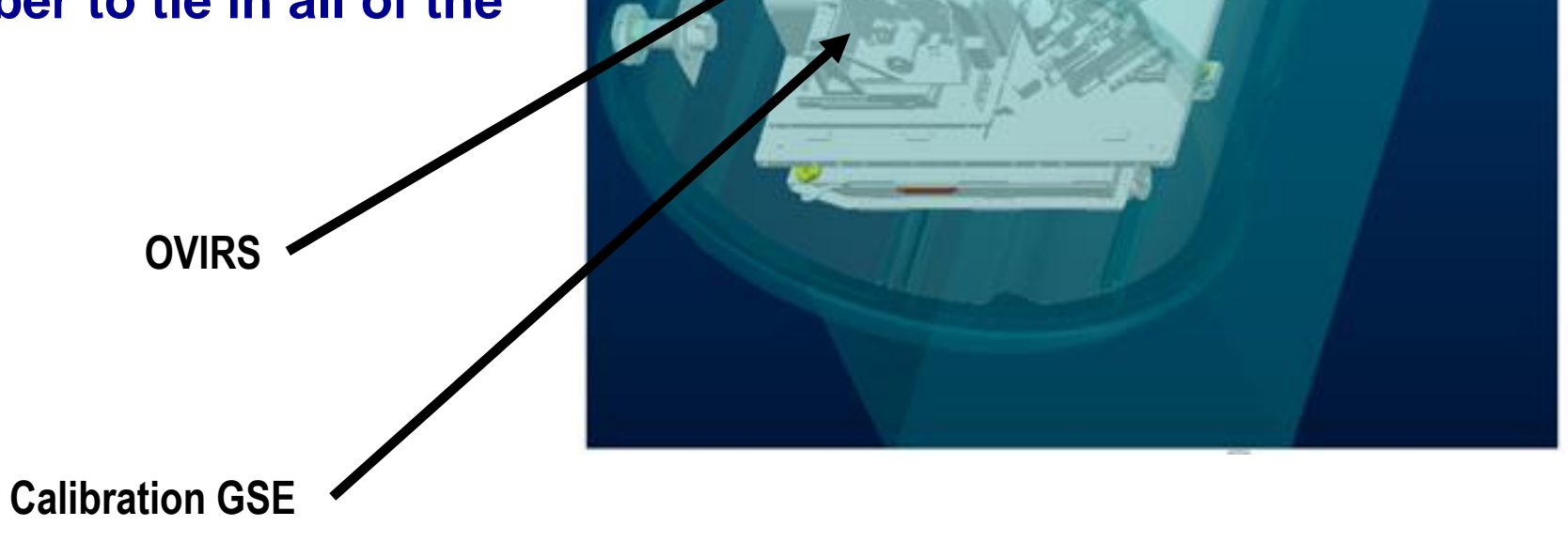


- Discussed some basic tools for measuring and aligning an optical system or instrument

- Large-volume metrology tools such as LR and LT can also be useful for small-scale optical alignment

- Metrology tools, references, techniques, and data reduction algorithms must be considered early during the planning phase of the development of an optical instrument or telescope or precision assembly

- Optical alignment is a broad subject, but we hope that this paper has added to the reader's toolbox a few ideas and techniques 


\author{
Phillip Coulter \\ Optics Branch \\ NASA Goddard Space Flight Center \\ Greenbelt, Maryland \\ Phillip.coulter@nasa.gov \\ tel. 3012869527
}




\section{Acknowledgements}

The author gratefully acknowledges the collective contributions of the

Engineers working on JWST and OSIRIS-REX and OVIRS.

This work is supported by the James Webb Space Telescope project at NASA Goddard Space Flight Center. 
Front view of the detector active area. Typical LR measurement spots are shown as circles.

Once the process below is complete the active area of the detector can be placed/aligned quickly into a system using the LR reference targets. Also any pixels position can be known via the LR reference targets and the results below

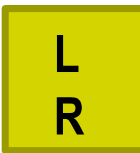

Process:

1. Laser radar (LR) measures the spherical reference targets

2. LR beam is pointed and focused at a location on the detectors active pixel area

3. A surface point measurement is made using the LR of beam location on the detector surface

4. The detector captures an image of the incident beam on the detector

5. Steps 1 through 4 are repeated while pointing the LR at several locations on the detectors active surface area. A minimum of 3 locations should be measured

6. The captured images are analyzed for the center of the beam at each location. The data is combined with the LR measurements. With multiple spots measured other pixel locations can be interpolated from the data
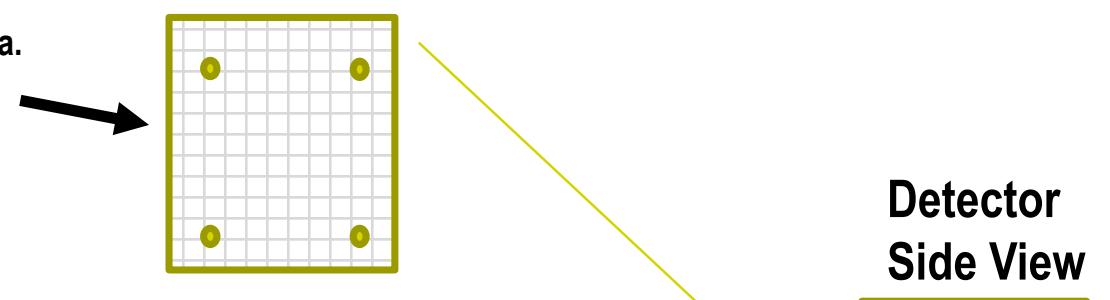

Side View

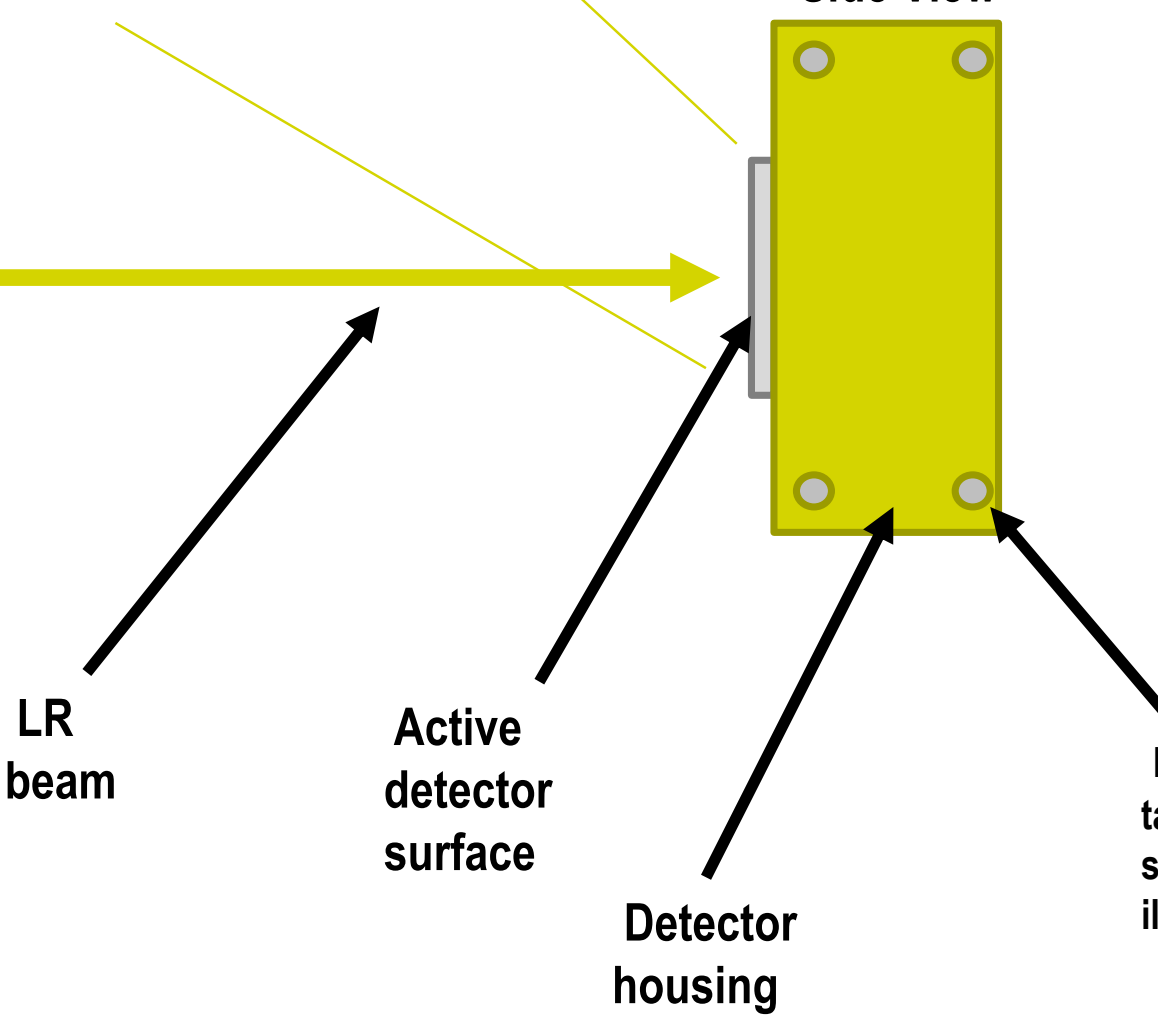

LR Reference targets (spheres) shown for illustrative purpose 


\title{
A Toolbox of Metrology-based Techniques for Optical System Alignment
}

\section{SPIE Optics \& Photonics}

\author{
San Diego, CA \\ August 28, 2016
}

hillip Coulter (GSFC), Peter' N. Blake (GSFC), Brent J. Bos (GSFC), Gordon V. Casto (GSFC), William L. Eichhorn (GESI), Jeffrey S. Gum (GSFC), Theodore J. Hadjimichael (GSFC), John G. Hagopian (JHEL), Joseph E. Hayden (SSC), \&amantha L. Hicks (GSFC), Dave Kubalak (GSFC), Kyle F. Mclean (PEMSL), Joseph McMann (SLI), Raymond G. Ohl (GSFC), Kevin Redman (SLI), Greg Wenzel (SLI), Jerrod Young (GSFC) 